ARTICLE

\title{
Robust but weak winter atmospheric circulation response to future Arctic sea ice loss
}

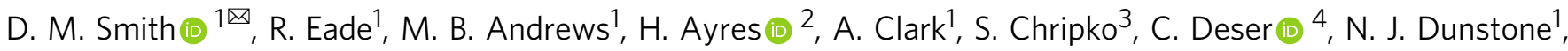 \\ J. García-Serrano ${ }^{5}$, G. Gastineau6 , L. S. Graff (10 7, S. C. Hardiman', B. He (i) ${ }^{8}$, L. Hermanson (1) 1, T. Jung (1) 9,10, \\ J. Knight ${ }^{1}$, X. Levine (i) ${ }^{11}$, G. Magnusdottir (i) ${ }^{12}$, E. Manzini ${ }^{13}$, D. Matei ${ }^{13}$, M. Mori (i) ${ }^{14}$, R. Msadek ${ }^{3}$, P. Ortega (i) ${ }^{11}$, \\ Y. Peings (1) ${ }^{12}$, A. A. Scaife (1) ${ }^{1,15}$, J. A. Screen (1) ${ }^{15}$, M. Seabrook ${ }^{1}$, T. Semmler (1) ${ }^{9}$, M. Sigmond (1) ${ }^{16}$, \\ J. Streffing 9,18, L. Sun $^{17} \&$ A. Walsh ${ }^{15}$
}

The possibility that Arctic sea ice loss weakens mid-latitude westerlies, promoting more severe cold winters, has sparked more than a decade of scientific debate, with apparent support from observations but inconclusive modelling evidence. Here we show that sixteen models contributing to the Polar Amplification Model Intercomparison Project simulate a weakening of mid-latitude westerlies in response to projected Arctic sea ice loss. We develop an emergent constraint based on eddy feedback, which is 1.2 to 3 times too weak in the models, suggesting that the real-world weakening lies towards the higher end of the model simulations. Still, the modelled response to Arctic sea ice loss is weak: the North Atlantic Oscillation response is similar in magnitude and offsets the projected response to increased greenhouse gases, but would only account for around $10 \%$ of variations in individual years. We further find that relationships between Arctic sea ice and atmospheric circulation have weakened recently in observations and are no longer inconsistent with those in models.

\footnotetext{
${ }^{1}$ Met Office Hadley Centre, Exeter, UK. ${ }^{2}$ Department of Meteorology, University of Reading, Reading, UK. ${ }^{3}$ CECI, Université de Toulouse, CNRS, CERFACS, Toulouse, France. ${ }^{4}$ National Center for Atmospheric Research, Boulder, CO, USA. ${ }^{5}$ Group of Meteorology, Universitat de Barcelona, Barcelona, Spain. ${ }^{6}$ UMR LOCEAN, Sorbonne Université/CNRS/IRD/MNHN, Institut Pierre Simon Laplace (IPSL), Paris, France. ${ }^{7}$ Norwegian Meteorological Institute, Oslo, Norway. ${ }^{8}$ State Key Laboratory of Numerical Modeling for Atmospheric Sciences and Geophysical Fluid Dynamics, Institute of Atmospheric Physics, Chinese Academy of Sciences, Beijing, China. ${ }^{9}$ Alfred Wegener Institute, Helmholtz Centre for Polar and Marine Research, Bremerhaven, Germany. ${ }^{10}$ Institute of Environmental Physics, University of Bremen, Bremen, Germany. ${ }^{11}$ Barcelona Supercomputing Center, Barcelona, Spain. ${ }^{12}$ Department of Earth System Science, University of California Irvine, Irvine, CA, USA. ${ }^{13}$ Max-Planck-Institut für Meteorologie, Hamburg, Germany. ${ }^{14}$ Research Institute for Applied Mechanics, Kyushu University, Fukuoka, Japan. ${ }^{15}$ College of Engineering, Mathematics and Physical Sciences, Exeter University, Exeter, UK. ${ }^{16}$ Canadian Centre for Climate Modelling and Analysis, Environment and Climate Change Canada, Victoria, BC, Canada. ${ }^{17}$ Department of atmospheric science, Colorado State University, Fort Collins, CO, USA. ${ }^{18}$ Present address: Jacobs University Bremen, Campus Ring 1, 28759 Bremen, Germany. ${ }^{凶}$ email: doug.smith@metoffice.gov.uk
} 
S ince the 1990s the Arctic has been warming more than twice as fast as the global average ${ }^{1}$, accompanied by rapid loss of sea ice ${ }^{2}$. This is consistent with polar amplification of climate change and is expected to continue in response to anthropogenic emissions of greenhouse gases ${ }^{3}$. Over the same period, winter temperatures over mid-latitude northern continents, especially Eurasia, have unexpectedly remained steady or cooled, with an apparent increase in severe winter weather ${ }^{4-7}$. The possibility that Arctic warming promotes more severe mid-latitude winters, by altering the atmospheric circulation, has been the subject of intense scientific debate ${ }^{4,5,8-19}$. Observational studies have suggested a clear link between Arctic sea ice loss and midlatitude winter severity $5,6,20-26$, but dedicated numerical model experiments, which are essential to establish causality and to understand the physical mechanisms, are inconclusive, with some simulating mid-latitude cooling in response to Arctic sea ice $\operatorname{loss}^{27-34}$ and others not supporting this link $\mathrm{k}^{16,18,35-40}$.

For Arctic warming to promote cooling over mid-latitudes would require changes in atmospheric circulation involving a weakening of mid-latitude westerly winds ${ }^{38}$, consistent with a negative phase of the North Atlantic Oscillation (NAO), and/or a strengthening of the Siberian High ${ }^{41,42}$. Hence, understanding and quantifying the midlatitude atmospheric circulation response to Arctic sea ice loss is critical, but there is currently little consensus in modelling studies: the full spectrum of NAO responses has been reported including negative $\mathrm{NAO}^{17,27,29,30,32,41,43,44}$, positive $\mathrm{NAO}^{45-50}$, little response $\mathrm{e}^{19,51-54}$ and a response that depends on the details of the forcing $28,31,36,55-58$ or the background state of the climate system ${ }^{59-61}$.

There are many potential reasons why previous modelling results are inconsistent, including the use of different magnitudes and patterns of imposed sea ice changes, treatment of oceanic feedbacks ${ }^{41}$, different models, and whether the simulated responses can be distinguished from internal variability. To overcome some of these limitations, the Polar Amplification Model Intercomparison Project ${ }^{62}$ (PAMIP) contribution to the sixth Coupled Model Intercomparison Project ${ }^{63}$ (CMIP6) proposed a set of coordinated experiments. Here, we analyse a large ensemble of PAMIP experiments consisting of more than 3000 simulations from 16 different models and find that all models simulate a weakening of mid-latitude tropospheric westerly winds in response to projected Arctic sea ice loss. We elucidate the main physical processes and show that the model spread depends on eddy feedback. This is 1.2 to 3 times too weak in the models, suggesting that the real-world weakening of westerly winds lies towards the higher end of the model simulations. We also show that observed relationships between Arctic sea ice and atmospheric circulation have weakened recently and are no longer inconsistent with those in models. However, the modelled response to Arctic sea ice loss is weak relative to inter-annual variability, though it is similar in magnitude and offsets the projected response to increased greenhouse gases.

\section{Results}

Multi-model response. The atmospheric response to future Arctic sea ice loss is diagnosed from two sets of global atmospheric model simulations (Methods). The first set (present-day) is driven by sea surface temperatures (SSTs) and sea ice concentrations (SICs) representing the present-day climate. The second set (future-Arctic) is the same except that Arctic sea ice and coincident SSTs are replaced with values expected if global temperatures rise by $2{ }^{\circ} \mathrm{C}$. By construction, the difference (future-Arctic minus present-day) provides the model-simulated response to future Arctic sea ice loss. We assess 16 model simulations each with between 98 and 300 ensemble members (Table 1) and forced with the same SSTs and SICs. We focus on the boreal winter season (December, January and February, DJF) for which the imposed sea ice changes show reductions around the edges of the ice pack, especially in the Barents-Kara Seas, Sea of Okhotsk, Bering-Chukchi Seas, and Hudson Bay and Labrador Sea (Fig. 1a). In winter, sea ice insulates the atmosphere from the warmer ocean. Hence warm SSTs are imposed where sea ice is lost in future, producing local maxima of near surface warming response in these regions along with further warming spread throughout the Arctic and into lower latitudes (Fig. 1b).

In the multi-model mean, there are minima in mean sea level pressure (MSLP) response situated over the regions of largest sea ice loss (Fig. 1c), consistent with a thermodynamic heat low response to surface warming. However, there is also a ridge of high pressure extending from Greenland to Siberia with low pressure further south, producing a response that projects onto a negative NAO and a strengthened Siberian High. Although these features are statistically significant in the multi-model ensemble mean (stippling in Fig. 1c), there is disagreement between individual models on the sign of the response in many regions (grey stars show where $90 \%$ of models agree).

Consistencies and differences among the models in the dynamical response are further illustrated in the zonally averaged zonal wind ( $\bar{u}$, where the overbar denotes the zonal average) response as a function of latitude and height (Fig. 2). In the troposphere, there is a very robust equatorward shift, with a weakening of zonal winds around $55-65^{\circ} \mathrm{N}$ and a strengthening around $30-40^{\circ} \mathrm{N}$ simulated by all models. However, the response is much less coherent in the stratosphere, with some models simulating a significant weakening but the majority showing an insignificant response of either sign. This suggests that a stratospheric pathway highlighted in some studies $5,30,33,64-66$ is not essential for the sign of the tropospheric and surface response. However, it could act to modulate the magnitude of the surface response, as discussed later. Even in the troposphere where the sign of the response is robust, regions of statistical significance (stippled in Fig. 2) are not consistent across models and the strength of the response varies greatly between models. This raises the key question of what the real-world response would be, and whether the differences between models can be understood in order to derive a constrained estimate. Further progress therefore requires understanding the physical processes.

Physical processes. We focus on explaining the robust multimodel average zonal-mean temperature and zonal wind responses in the troposphere. The largest zonal-mean warming $(\bar{T})$ occurs close to the surface over the Arctic (Fig. 3a), consistent with direct heating of the atmosphere by the imposed local surface warming associated with the loss of sea ice. There is a second warming maximum over the Arctic in the lower stratosphere (centred around $100-200 \mathrm{hPa}$ ) which has previously been suggested to be heated directly by energy transported from below ${ }^{5}$. However, in the model simulations there is a meridional overturning circulation response with air descending at high latitudes in the lower stratosphere (arrows in Fig. 3a) indicating that this region warms adiabatically as part of the dynamical response ${ }^{67}$. Furthermore, warming in this region is not robust across the models. Hence, the robust weakening of the mid-latitude tropospheric winds is unlikely to be solely caused by a simple reduction of meridional temperature gradient by heating of the high latitude atmosphere from below.

A meridional overturning circulation response is also seen in the mid-latitude lower troposphere (Fig. 3a) and, as we show below, is important for understanding the physical mechanisms and explaining the spread in modelled responses. This circulation is thermally indirect, with air rising over relatively cool surface 


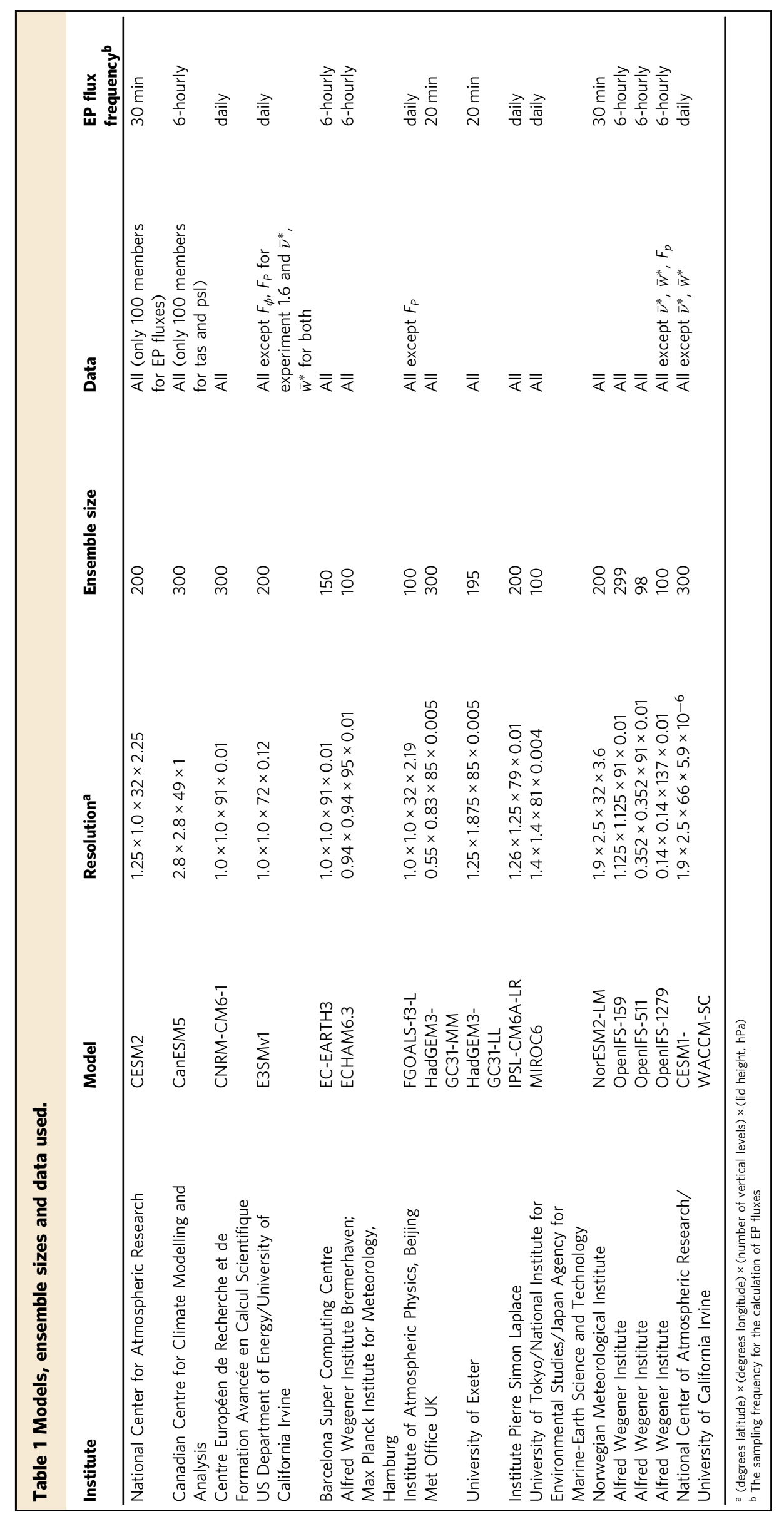


(a) SIC

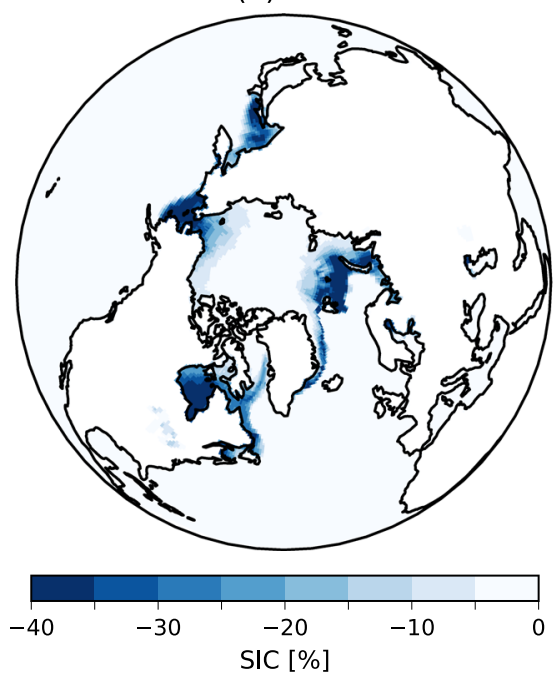

(b) TAS

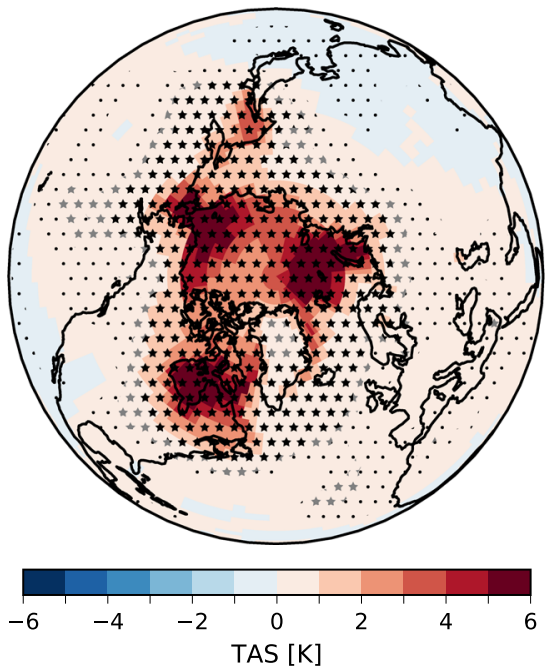

(c) MSLP

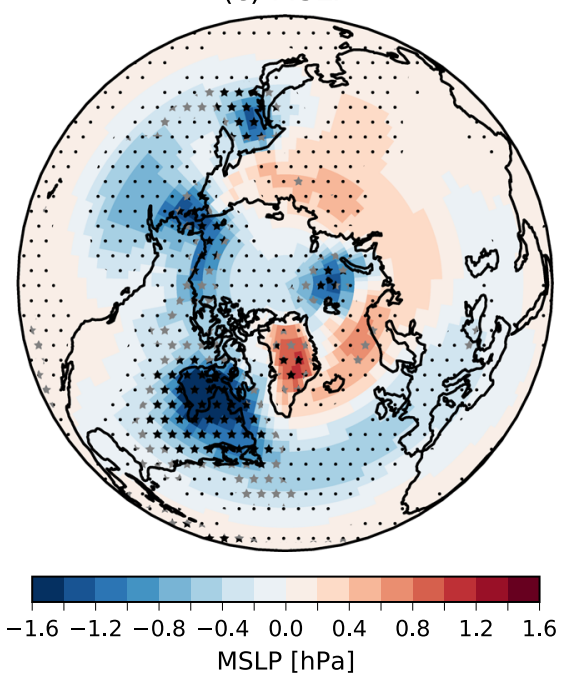

Fig. 1 Winter response to future Arctic sea ice loss. a Imposed winter sea ice concentration difference (\%). b Near surface temperature (TAS) response (K). Note that surface temperature changes are imposed in regions of sea ice loss. c Mean sea level pressure (MSLP) response (hPa). All plots show the winter (December, January, February, DJF) mean, and responses are for the multi-model ensemble mean (calculated as the unweighted average of all ensemble members). Stippling indicates where the multi-model ensemble mean response is significant ( $95 \%$ confidence interval). Black (grey) stars indicate where 100\% (90\%) of the individual models agree on the sign of the response.

conditions between $35-50^{\circ} \mathrm{N}$ and descending over the warmer surface between 55 and $70^{\circ} \mathrm{N}$. It is therefore not a direct thermally driven response to the imposed high latitude warming and, as we show below, highlights a key role for changes in wave (eddy) activity.

Climatologically, transient wave activity is mostly generated near the surface by baroclinic eddies in the storm tracks around $40-60^{\circ} \mathrm{N}$ and propagates vertically and meridionally until the waves break and dissipate ${ }^{68}$. Waves flux angular momentum in the opposite direction to their propagation, accelerating zonal winds in their source regions and decelerating zonal winds in their dissipation regions. Wave propagation may be depicted by Eliassen-Palm (EP) fluxes (Methods equation 6), with regions of EP flux divergence indicating where waves act to accelerate the zonal flow and regions of EP flux convergence indicating where waves act to decelerate the zonal flow (Methods equations 2 and 5).

The multi-model mean wave activity response (Fig. 3c) mainly consists of an equatorward shift of upward EP flux $\left(F_{p}\right)$ in the troposphere, with increased $F_{p}$ around $35-55^{\circ} \mathrm{N}$ and decreased $F_{p}$ around $60-75^{\circ} \mathrm{N}$, and an increased northward EP flux $\left(F_{\phi}\right)$ in the mid to upper troposphere connecting these latitudes. The resulting divergence of $F_{\phi}$ accelerates the zonal wind around $30-40^{\circ} \mathrm{N}$ and convergence of $F_{\phi}$ decelerates the zonal wind around $55-65^{\circ} \mathrm{N}$ (Fig. 3d), consistent with the robust equatorward shift of the winds noted above. We will show below that the strength of the EP flux response is important for explaining the model spread, and focus first on understanding its origins.

Several previous studies ${ }^{30-32,36,69-71}$ have highlighted an increase in $F_{p}$ in response to Arctic sea ice loss. However, the cause is unclear given that the reduced meridional temperature gradient between the equator and the pole (Fig. 3a) would weaken the baroclinic generation of eddies in the storm tracks ${ }^{72,73}$ and hence reduce $F_{p}$, as seen in other studies ${ }^{43,61,67,74}$. There is a small increase in $F_{p}$ north of $80^{\circ} \mathrm{N}$ (Fig. 3c) that is consistent with zonal asymmetries in near surface temperature and sea level pressure response over regions of sea ice loss (Fig. 1), but the main signal is an equatorward shift resulting in an increase in $F_{p}$ around $35-55^{\circ} \mathrm{N}$ that also extends into the stratosphere. Hence, understanding the main physical processes involved in this equatorward shift is key for understanding the midlatitude atmospheric response to Arctic sea ice loss.
Although causality cannot be unequivocally determined, much insight can be gained by considering the evolution from autumn to winter, focussing on processes that are robustly simulated across the models. In October there is a weakening of both $\bar{u}$ and $F_{p}$ around $50-75^{\circ} \mathrm{N}$ (Fig. $4 \mathrm{~b}, \mathrm{c}$ ) consistent with the imposed reduction in meridional surface temperature gradient, whereas an equatorward shift is first seen in November (Fig. 4f, g) and develops into the DJF pattern (Fig. 3). To explain this evolution we highlight the following processes which appear to be simulated by the majority of models, though we note that they do not explain every aspect of the circulation response and other processes ${ }^{75}$ are also likely operating:

1. Reduced zonal wind shear and eddy formation. Arctic warming decreases the surface meridional temperature gradient in October (Fig. 4a), reducing the wind shear on the poleward side of the jet (around $60-70^{\circ} \mathrm{N}$, Fig. $4 \mathrm{~b}$ ) via the thermal wind relation (Methods equation 1). Reduced wind shear reduces baroclinic eddy formation, weakening the storm track and reducing $F_{p}$ at the surface around $50-75^{\circ} \mathrm{N}$ (Fig. 4c).

2. Meridional overturning circulation. A lower tropospheric mid-latitude meridional overturning circulation anomaly (Fig. 4a, e, highlighted above) develops between October and December such that the resulting flow maintains thermal wind balance and is consistent with changes in eddy activity. Some aspects of this circulation can be understood by considering that the reduced $F_{p}$ at the surface around $50-75^{\circ} \mathrm{N}$ (Fig. $4 \mathrm{c}, \mathrm{g}$ ) results in a positive $\nabla_{p} F_{p}$ immediately above (Fig. $4 \mathrm{~d}, \mathrm{~h}$ ) because the flux into this region reduces more than the flux out of this region. An increase in $\nabla_{p} F_{p}$ must be balanced (Methods equation 2) by an increase in zonal wind and/or an equatorward flow (negative $\bar{v}^{*}$ ). However, zonal wind tends to be reduced in response to the imposed weakening of the surface temperature gradient (Fig. 4b) and $\nabla_{p} F_{p}$ is at least partly balanced by an equatorward flow near the surface around $45-55^{\circ} \mathrm{N}$ (Fig. $4 \mathrm{a}$, e). To maintain mass continuity, a meridional circulation develops with ascent further equatorward $\left(35-50^{\circ} \mathrm{N}\right)$, poleward flow in the mid to upper troposphere $\left(50-70^{\circ} \mathrm{N}\right)$ and descent around $65-75^{\circ} \mathrm{N}$. 

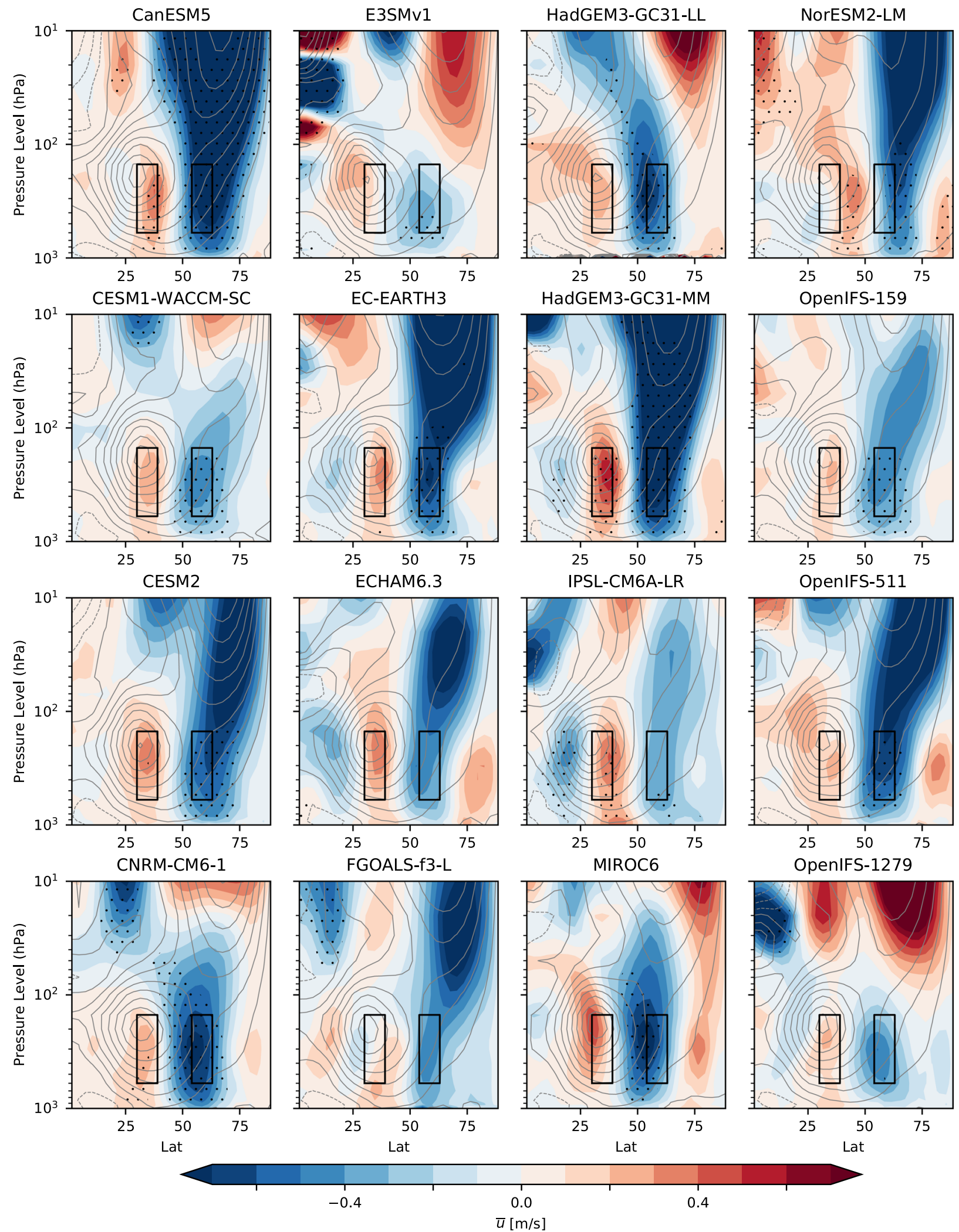

Fig. 2 Consistent tropospheric response to Arctic sea ice loss. Zonally averaged DJF zonal wind response ( $\bar{u}$, $\mathrm{ms}^{-1}$, where the overbar denotes the zonal average) plotted as a function of latitude $\left({ }^{\circ} \mathrm{N}\right)$ and height (pressure) for the ensemble mean of each of the models. The boxes show the regions used to compute the zonal wind response index (ZWRI). Stippling indicates where the ensemble mean response is significant ( $95 \%$ confidence interval). Contours show the climatological zonal mean winds (contour interval $5 \mathrm{~ms}^{-1}$ with negative contours dotted). 
(a) $\bar{T}$

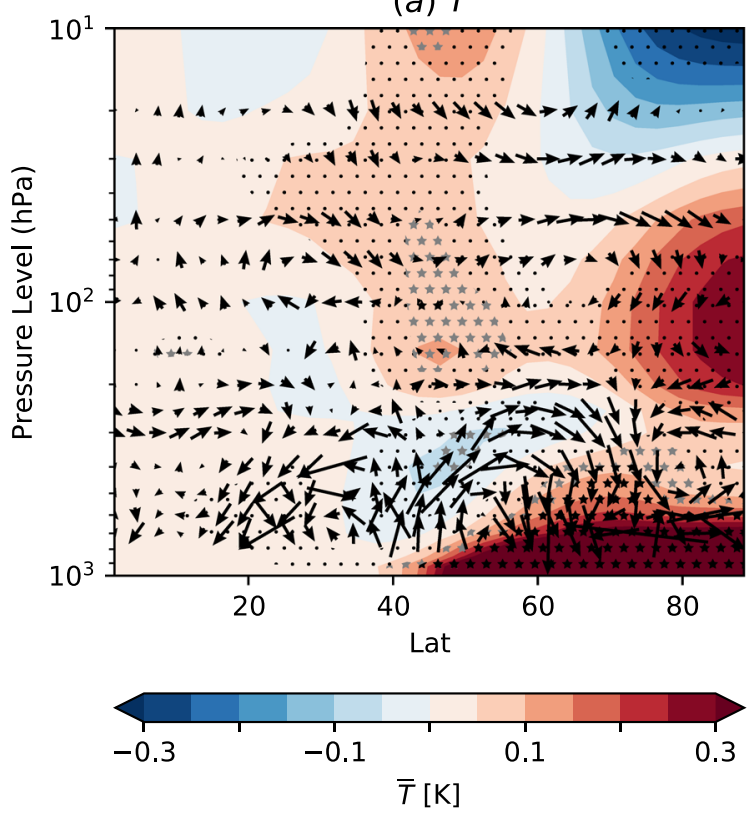

(c) $F_{p}$

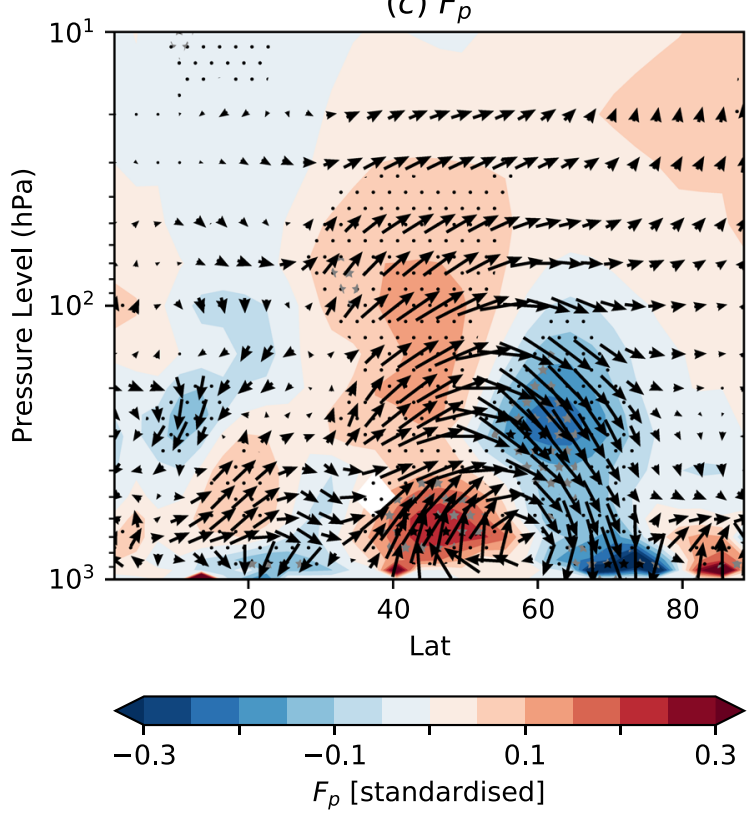

(b) $\bar{u}$
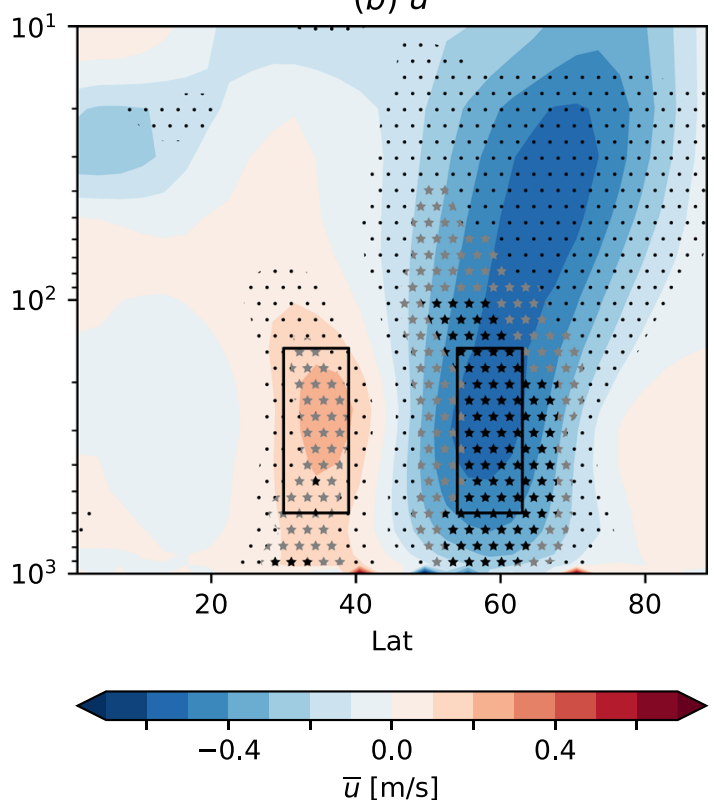

(d) $\nabla_{\phi} F_{\phi}$

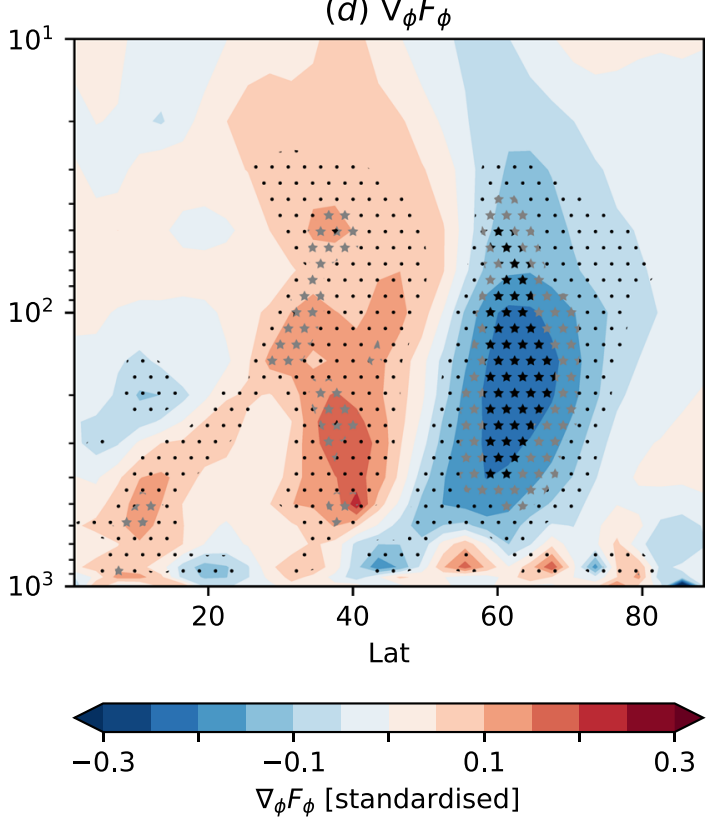

Fig. 3 Zonal mean response. Latitude-height cross sections of the multi-model ensemble mean zonally averaged DJF response of a temperature ( $\bar{T}$, colours, $K$, where the overbar denotes the zonal average) and transformed Eulerian mean (TEM, Methods) circulation (arrows), b zonal wind $\left(\bar{u}, m s^{-1}\right)$, and c upward Eliassen-Palm (EP) flux ( $F_{p}$, colours, standard deviations) and EP flux vectors (arrows representing $F_{\phi}$ and $F_{p}$ ), d divergence of northward EP flux $\left(\nabla_{\phi} F_{\phi}\right.$, standard deviations). Stippling as in Fig. 1. To aid visualisation the TEM circulation and EP fluxes are standardised by dividing by the internal variability of the present-day simulations (Methods).

Adiabatic cooling of the ascending air around $35-50^{\circ} \mathrm{N}$ would tend to enhance the meridional temperature gradient and, via thermal wind, strengthen the wind shear further to the south, and reduce the meridional temperature gradient and weaken the wind shear further to the north. Hence, this meridional circulation tends to promote an equatorward shift of the storm track and source of $F_{p}$, further reducing $F_{p}$ on the poleward side of the jet and producing an increase in $F_{p}$ on the equatorward side as seen in the DJF pattern of wave activity response (Fig. 3c).

3. Positive eddy feedback. The wave activity response (Fig. 3c) results in a divergence of $F_{\phi}$ on the equatorward side of the jet and convergence of $F_{\phi}$ on the poleward side of the jet (Fig. 3d) that reinforces the equatorward shift of the storm track and hence enhances the response. Increased $F_{p}$ can also propagate into the stratosphere, weakening the polar vortex and subsequently further enhancing the equatorward shift by weakening the tropospheric winds on the poleward side of the jet, especially in late winter.

Sensitivity across models. We further assess the processes described above by investigating whether they explain the sensitivity of the response across the models. To quantify the response 


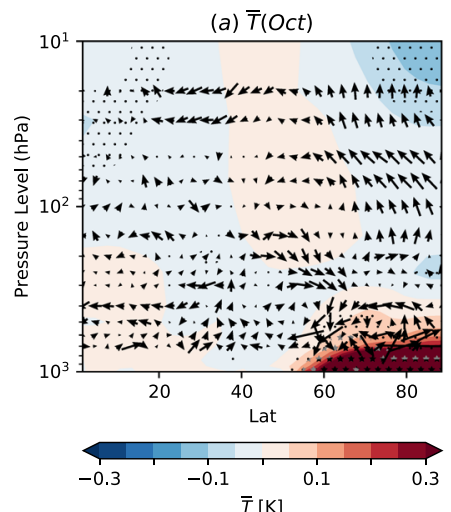

(c) $F_{p}(O c t)$

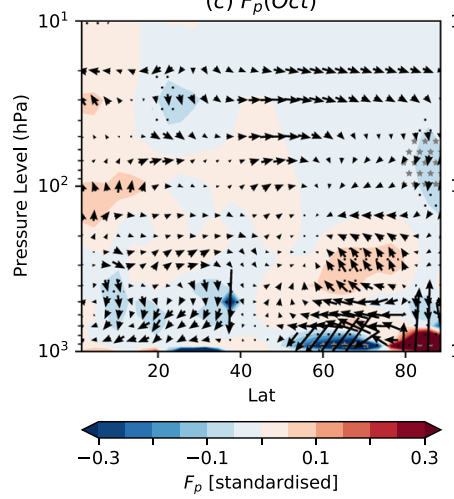

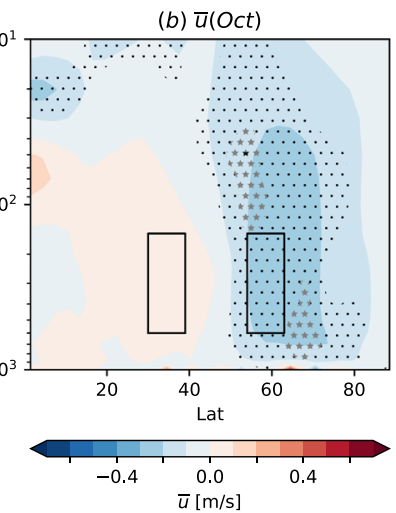

(d) $\nabla_{p} F_{p}($ Oct $)$

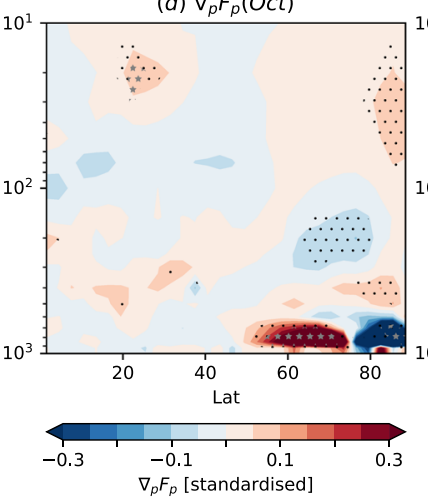

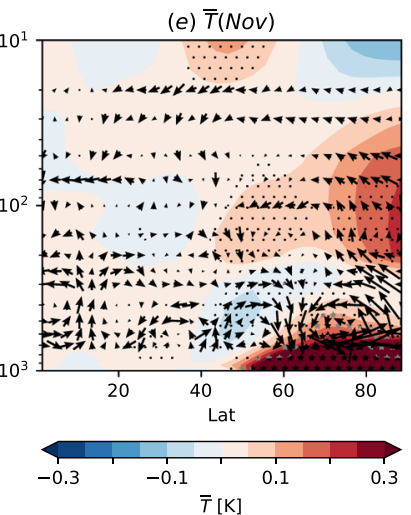

(g) $F_{p}($ Nov $)$

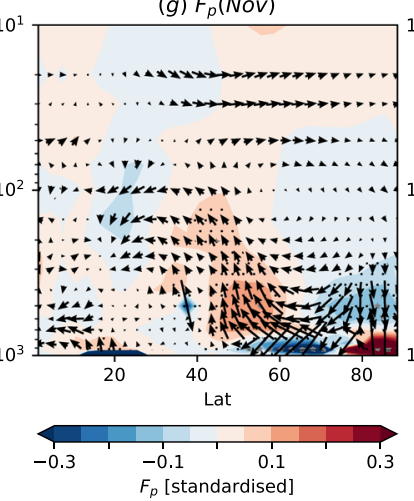

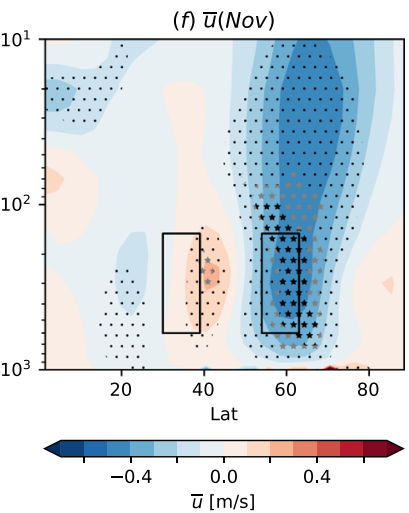

(h) $\nabla_{p} F_{p}($ Nov $)$

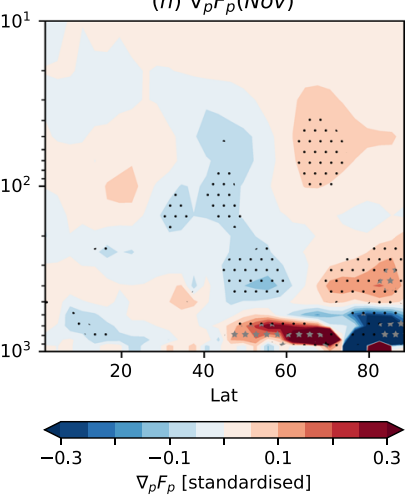

Fig. 4 Physical mechanisms of zonal mean response. Latitude-height cross sections of the multi-model ensemble mean zonally averaged October

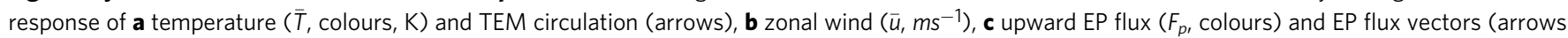
representing $F_{\phi}$ and $\left.F_{p}\right)$, d divergence of upward EP flux $\left(\nabla_{p} F_{p}\right)$. (e,f,g,h) As $(\mathbf{a}, \mathbf{b}, \mathbf{c}, \mathbf{d})$ but for November. Stippling as in Fig. 1 . To aid visualisation the TEM circulation and EP fluxes are standardised by dividing by the internal variability of the present-day simulations (Methods).

(a) $\operatorname{corr}(Z W R I, \bar{T})$

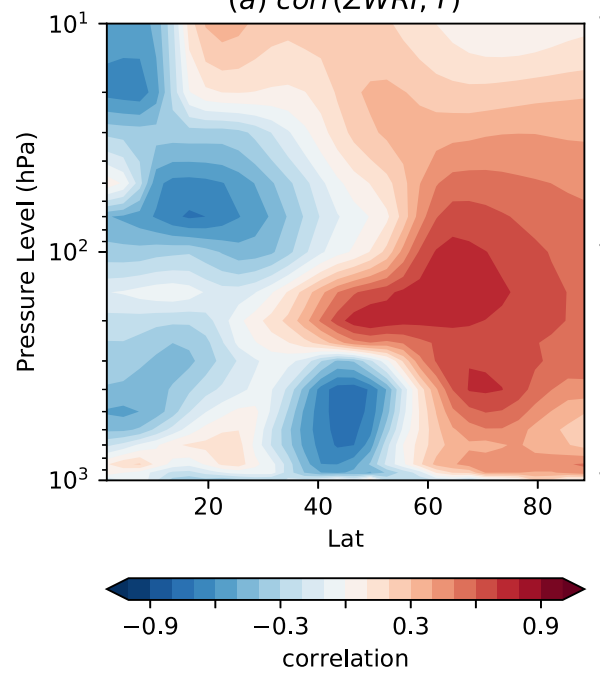

(b) $\operatorname{corr}\left(Z W R I, \nabla_{\phi} F_{\phi}\right)$

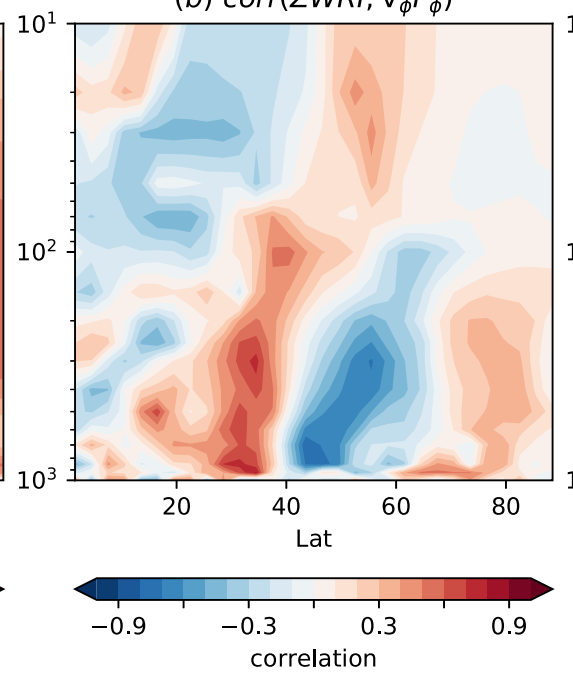

(c) $\operatorname{corr}\left(Z W R I, n^{2}\right)$

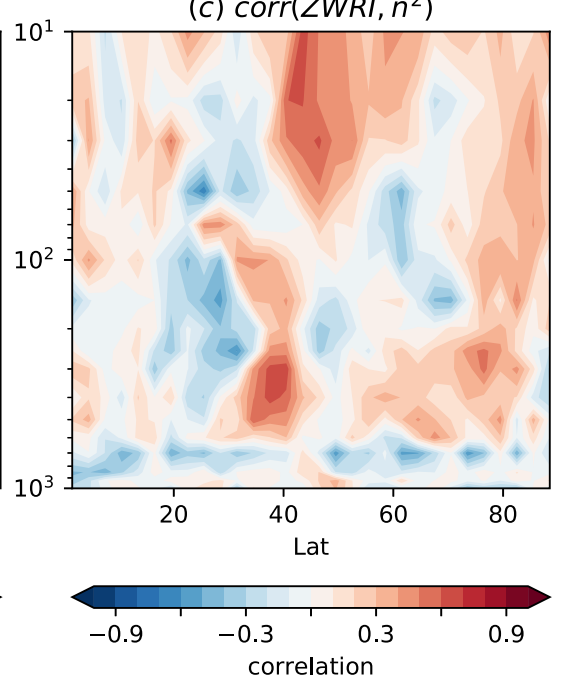

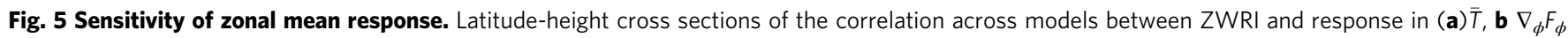
and $\mathbf{c}$ refractive index $\left(n^{2}\right)$. All data are for DJF.

for each model, we define a zonal wind response index (ZWRI, Methods, the difference between the zonally averaged zonal wind responses in the boxes in Figs. 2 and 3b). The correlation between ZWRI and zonal mean temperature response across the models (Fig. 5a) is consistent with a sensitivity to the strength of the meridional circulation (process 2, Fig. 3a): models with a larger ZWRI show enhanced lower tropospheric adiabatic cooling by ascent around $35-50^{\circ} \mathrm{N}$ and warming by descent around $65-75^{\circ} \mathrm{N}$ compared to models with a smaller ZWRI. In addition, models with a larger ZWRI show greater mid to upper tropospheric warming due to enhanced southerly advection around $50-70^{\circ} \mathrm{N}$ compared to models with a smaller ZWRI.

ZWRI is also correlated with the divergence of $F_{\phi}$ around $30-40^{\circ} \mathrm{N}$ and convergence of $F_{\phi}$ around $45-65^{\circ} \mathrm{N}$ (Fig. 5b) consistent with a positive feedback from wave driving in determining the magnitude of the simulated response (process 3 ). The tropospheric response may also be enhanced by eddy feedback involving the stratosphere (process 3): increased $F_{p}$ enters the stratosphere around $35-55^{\circ} \mathrm{N}$ 
(a) $\operatorname{Corr}\left(\bar{u}, \nabla_{\phi} F_{\phi}\right)$

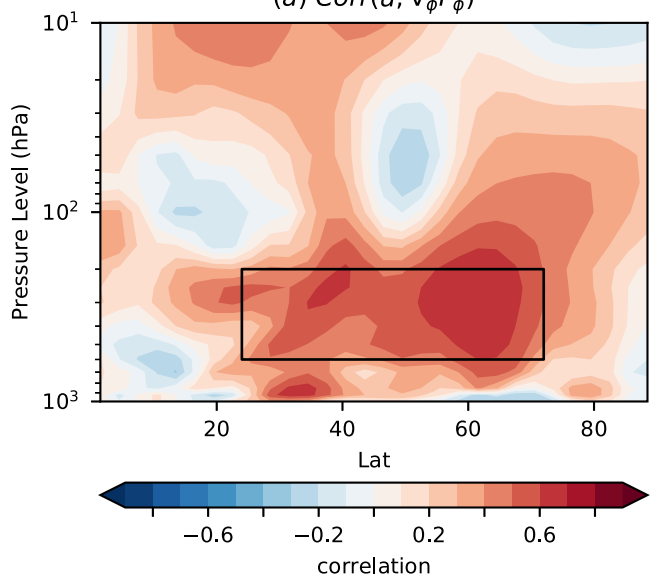

(b) Eddy Feedback

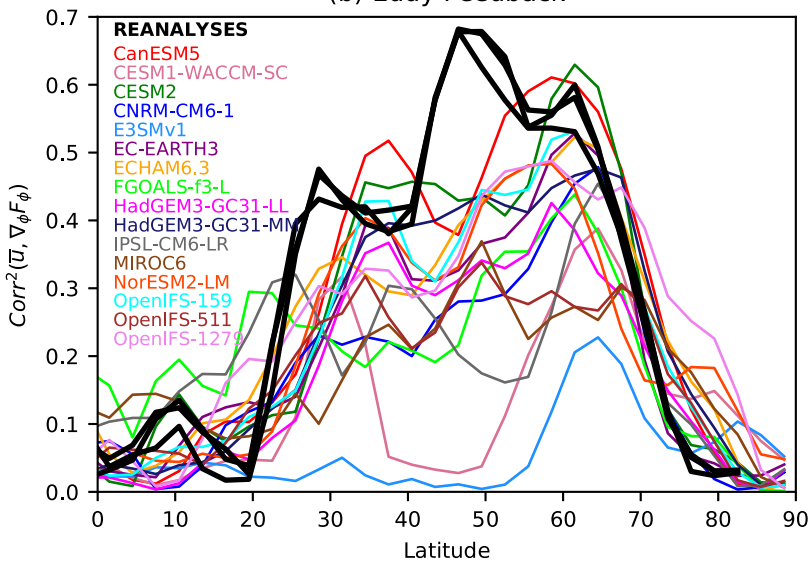

Fig. 6 Eddy feedback. a Latitude-height cross section of the multi-model mean local correlation between DJF $\bar{u}$ and $\nabla_{\phi} F_{\phi}$. Correlations are computed across ensemble members for the present day simulation for each model separately, and then averaged to make the multi-model mean. $\mathbf{b}$ Fraction of midupper troposphere zonal wind variance explained by $\nabla_{\phi} F_{\phi}$ as a function of latitude (the square of the local correlations in a averaged over 600 to 200 hPa) for each of the models (coloured curves) and in the reanalyses (black curves). Reanalyses values are computed from interannual time series over the period 1979-2016 inclusive. The eddy feedback parameter (M) is computed as the average over latitudes $25-72^{\circ} \mathrm{N}$ (shown by the box in a).

(Fig. 3c) and ZWRI is correlated with increases in the refractive index $\left(n^{2}\right.$, Methods equation 7) in these latitudes (Fig. 5c), allowing more waves to weaken the stratosphere and affect the troposphere via downward propagation, strengthening the negative $\mathrm{NAO}$ and equatorward jet shift through further eddy feedback ${ }^{76}$.

Constrained response. The model spread can potentially be exploited to obtain an estimate of the true response using the concept of emergent constraints (Methods). In this approach, the key physical processes that explain the differences between modelled responses must be understood and related to quantities that can be observed. If a robust relationship exists, then the true response may be inferred by comparing observations of these quantities with those in the models. Results above suggest an important role for eddies: the DJF zonal wind response is initiated by thermal wind balance but is sensitive to eddy feedback which alters $\nabla_{\phi} F_{\phi}$ in the troposphere (Figs. $3 \mathrm{~d}$ and $5 \mathrm{~b}$ ) and potentially involves the stratosphere via changes in $F_{p}$ and $n^{2}$ (Figs. $3 c$ and $5 \mathrm{c}$ ). Eddies are generated by the mean flow but can also feedback onto the mean flow in such a way that increases or reduces their generation (i.e. a positive or negative feedback ${ }^{77,78}$ ). Hence, we hypothesise that the response to sea ice loss may be related to the strength of the eddy feedback simulated by the different models.

We estimate eddy feedback by examining the role of eddies in driving internal variability (Methods). For each model, we compute the local correlation across the ensemble members between DJF zonal mean zonal wind $(\bar{u})$ and the divergence of northward EP flux $\left(\nabla_{\phi} F_{\phi}\right)$ in the present-day simulations. Averaged across the models, this correlation is positive throughout most of the troposphere (Fig. 6a) consistent with acceleration of the mean flow by a convergence of eddy momentum flux. The square of this correlation represents the fraction of the total variability of $\bar{u}$ that is explained by variations in $\nabla_{\phi} F_{\phi}$ and it would be expected to increase as the eddy feedback becomes more strongly positive. The squared correlation varies greatly across the models (Fig. 6b), suggesting a wide range of eddy feedbacks simulated by the different models.

We define a measure of eddy momentum feedback strength (M) as the variance of DJF $\bar{u}$ explained by $\nabla_{\phi} F_{\phi}$ (i.e. the squared correlation) averaged over the mid to upper extratropical troposphere in the region shown by the box in Fig. 6a (Methods). We find that $\mathrm{M}$ is positively correlated with ZWRI across models
( $r=0.49, p=0.03$, Fig. 7a), supporting our hypothesis that the response to sea ice loss is strengthened by eddy feedbacks and allowing a constrained estimate of the zonal wind response to be obtained via the ensemble regression approach (ER, Methods). We diagnose the observed eddy feedback using three reanalyses and computing the correlations in time rather than across ensemble members (Methods). The observed eddy feedback is between 1.2 and 3 times larger than that in any of the climate models. Using the observed eddy feedback strength to scale the zonal wind responses in the models, we find that the multi-model ensemble mean ZWRI increases from $0.7( \pm 0.1) \mathrm{ms}^{-1}$ to $0.9( \pm 0.2) \mathrm{ms}^{-1}$ ( $95 \%$ confidence intervals) (Fig. 7a, green shading), suggesting that the real world response would lie towards the higher end of the model simulations.

We now apply the ER method in a more general sense to assess the response to sea ice loss in different quantities and regions: for any variable and location, we regress our estimate of eddy momentum feedback strength against the response in that variable and use the observed eddy feedback to diagnose the constrained response. Note, that ER will have little impact where the regression is small. We find that ER enhances the zonal wind response throughout the atmosphere, including the stratospheric polar vortex (Fig. $7 b, r=-0.44, p=0.04$ ) which increases in magnitude from 0.1 to $-0.6 \mathrm{~ms}^{-1}$ in the simple ensemble mean (EM) to -0.1 to $-1.7 \mathrm{~ms}^{-1}$ in ER, accompanied by an increase in regions showing a significant zonal wind response (compare Fig. 8c, d). This is consistent with an increased refractive index response in ER particularly around $35-40^{\circ} \mathrm{N}$ (Fig. 5c) in the upper troposphere, which allows more waves to propagate into the stratosphere and weaken the SPV (Fig. 8a, b). It is well established that changes in the stratosphere propagate downwards and affect the troposphere and surface winds ${ }^{76}$, and this stratospheric pathway likely enhances the near surface wind response. Overall, ER results in a greater reduction of the SPV (Fig. 8c, d), stronger equatorward shift of the storm tracks (Fig. 8e, f), and a stronger negative NAO response (increased from -0.4 to $-1.2 \mathrm{hPa}$ in EM to -0.3 to $-2.1 \mathrm{hPa}$ in ER). However, ER does not constrain the Eurasian temperature response (Eurasia T, Methods), which ranges from -0.2 to $+0.4^{\circ} \mathrm{C}$.

We assess the robustness of our proposed constraint to several sources of uncertainty. The simulated response to Arctic sea ice loss is small relative to internal variability, leading to substantial uncertainties in individual models (ellipses in Fig. 7). 
(a) Constrained troposphere response

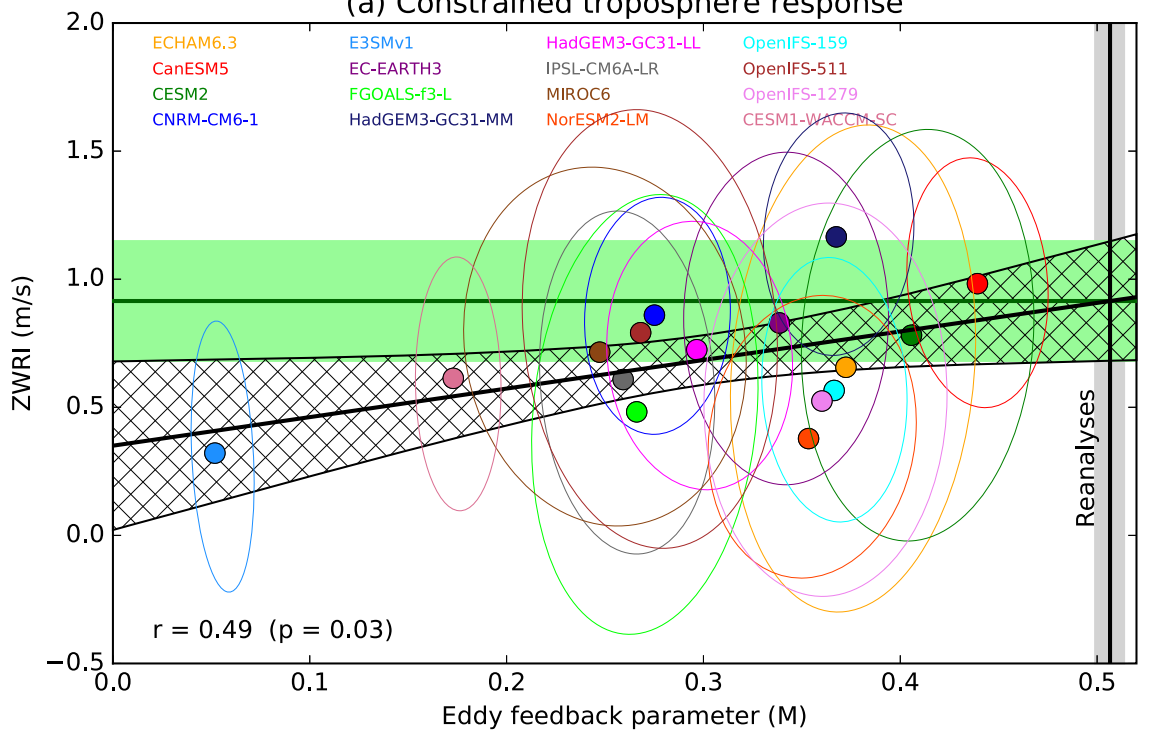

(b) Constrained stratosphere response

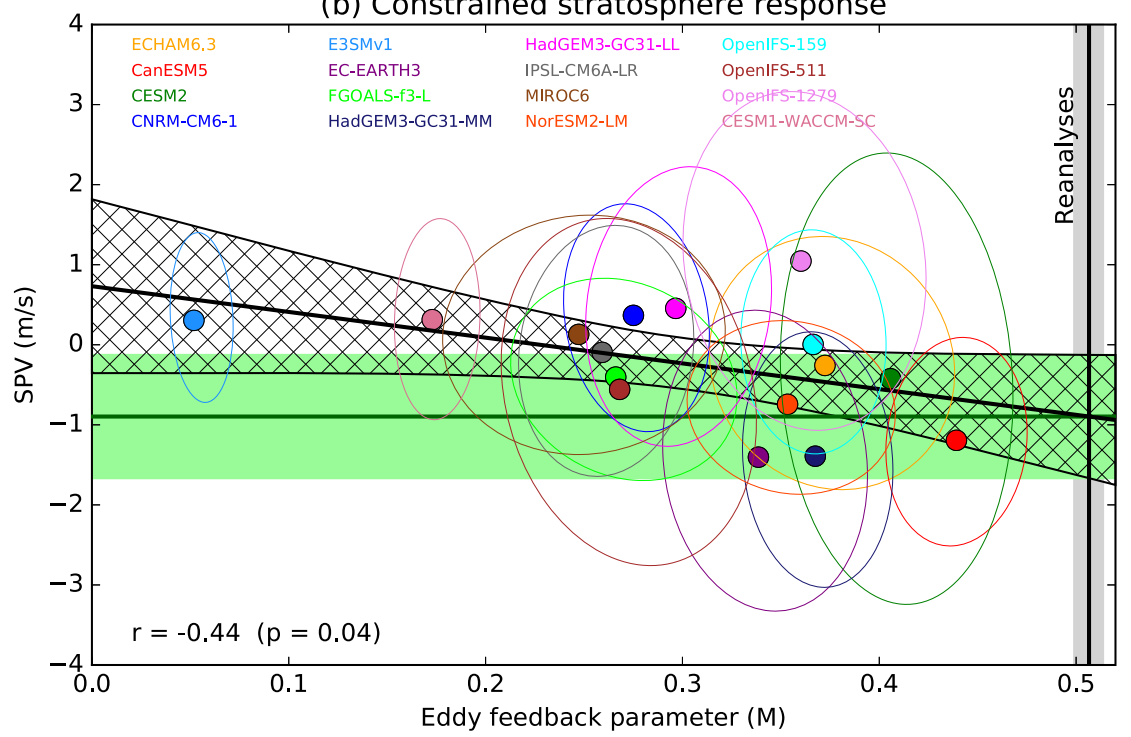

Fig. 7 Emergent constraints. a Emergent constraint based on the ensemble regression (ER) between eddy momentum feedback and the zonal wind response index (ZWRI). Black line shows the regression with hatching showing the $95 \%$ confidence interval. Horizontal green line shows the constrained ensemble mean response, with the shading showing its $95 \%$ confidence interval (Methods). Vertical black line and grey shading shows the mean and range of eddy feedback from the reanalyses. Ellipses show the $95 \%$ uncertainties obtained by bootstrapping with replacement the ensemble members. b As a but for the stratospheric polar vortex (SPV) response. A one-sided test is used to calculate $\mathrm{p}$ values since we expect the response to increase as eddy feedback strengthens. All data are for DJF.

We therefore further assess the statistical significance of the regressions by bootstrapping the individual members for each model with replacement. This is repeated 1000 times and results in $p$ values that are slightly less significant $(p=0.08$ and 0.06 for ZWRI and SPV respectively), highlighting the need for very large ensembles to obtain robust results ${ }^{79}$. We also tested the sensitivity of ER to outliers ${ }^{80}$ by removing each model in turn and repeating the regression. This is most sensitive to removing E3SMv1 and CanESM5, which increases the $p$ values to 0.16 and 0.07 respectively for ZWRI, and to 0.06 and 0.10 respectively for SPV. The calculation of EP fluxes can be sensitive to the frequency of data used, which ranges from 20 min to daily means depending on the model (Table 1 ) and $6 \mathrm{~h}$ in the reanalyses ${ }^{81}$. We tested this sensitivity by recalculating the regressions (Fig. 7) for subsets of models with similar sampling frequencies (20-30 min, 6 hourly, and daily) and found the sign to be the same for each subset as for the full set (though the regressions were no longer significant at the $95 \%$ confidence level). The eddy feedback parameters computed from the Reanalyses are based on 38 years compared to more than 100 members for the models. However, the three reanalyses are in good agreement and together provide a similar sample size to the models. Finally, the eddy feedback parameters for reanalyses are based on time series that include the effects of coupled modes of internal variability such as El Niño, in addition to changes in natural and anthropogenic radiative forcings that are not present in the model experiments. We recalculated the eddy feedback using time series from AMIP simulations that also include these factors for five models (CanESM5, HadGEM3-LL, HadGEM3-MM, IPSL-CM6A-LR, MIROC6) and found a small increase (0.09 on average, compared to a multi-model mean of 0.30 ), but that all model values remain lower than the reanalyses. 

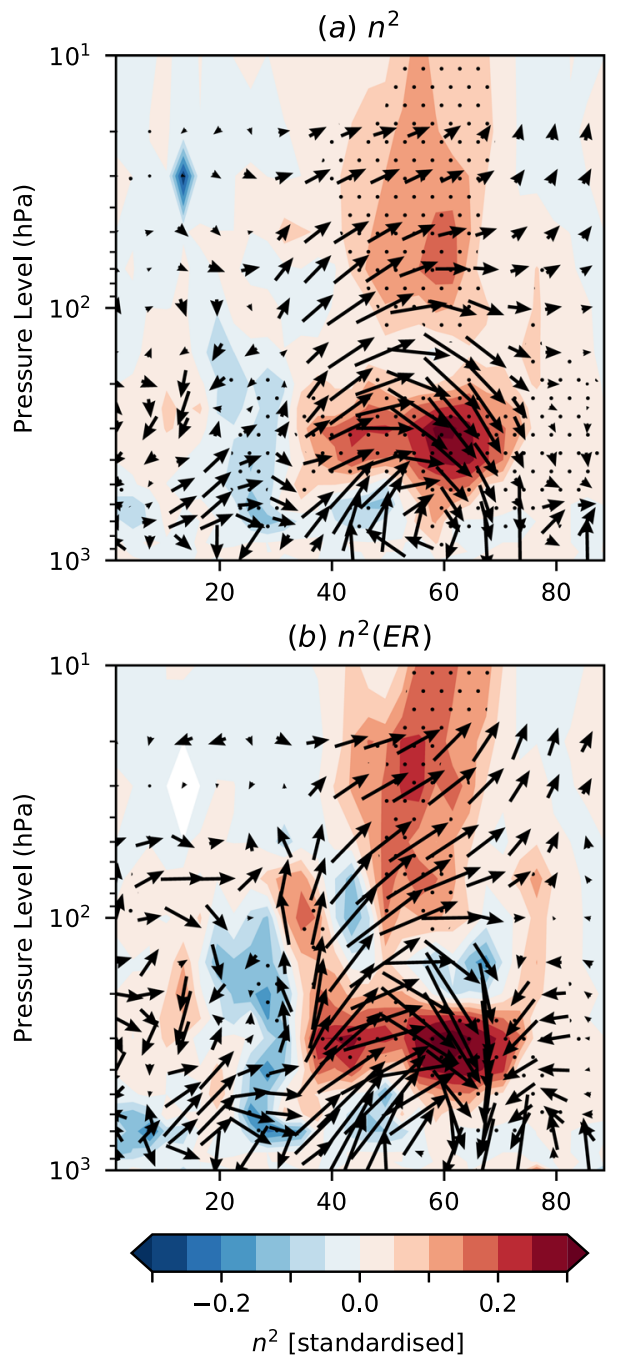
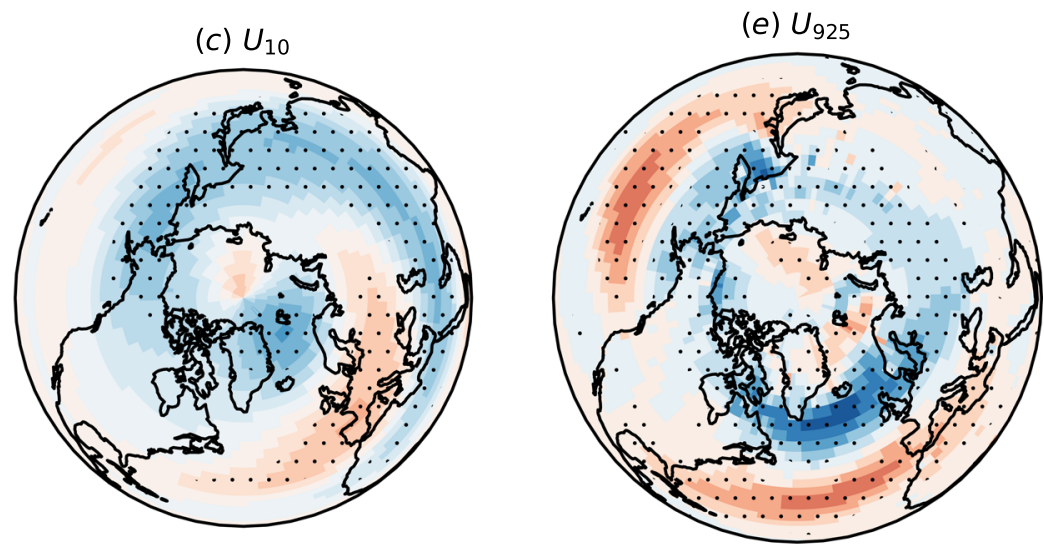

(d) $U_{10}(E R)$
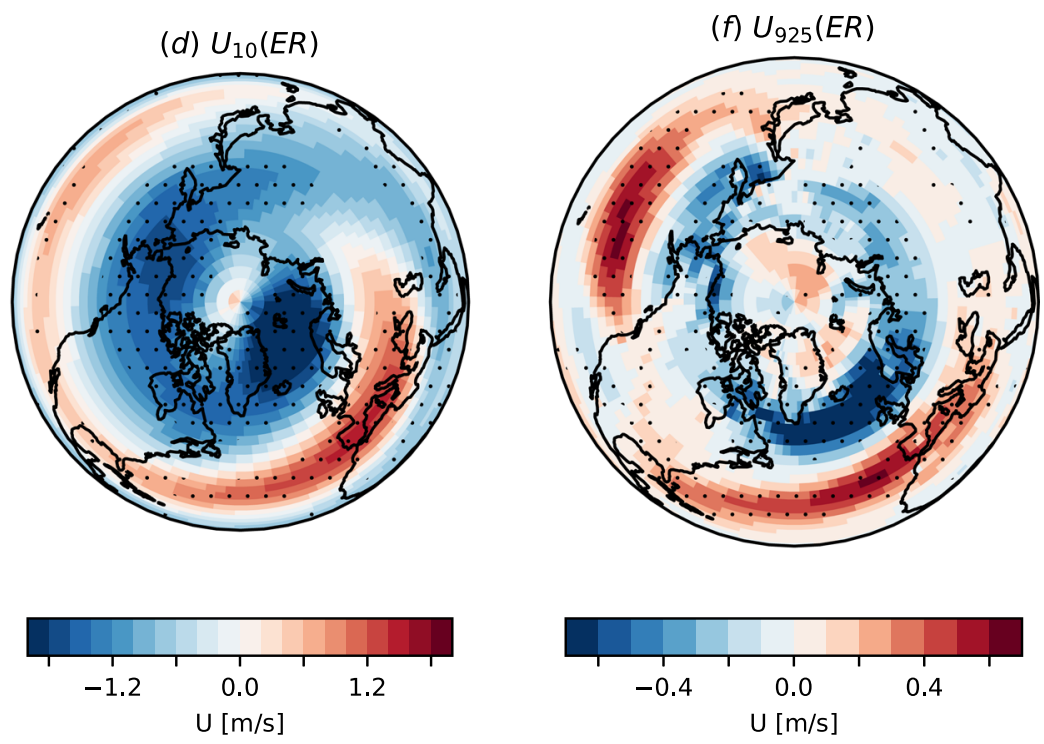

Fig. 8 Impact of emergent constraint. Refractive index $\left(n^{2}\right)$ response (standardised) for a the multi-model ensemble mean (EM) and $\mathbf{b}$ the ensemble regression (ER). c, d As a, b but for stratospheric zonal wind ( $m s^{-1}$ ) at $10 \mathrm{hPa}$. e, f As a, b but for near-surface zonal wind (ms ${ }^{-1}$ ) at $925 \mathrm{hPa}$. Arrows in $\mathbf{a}$ and $\mathbf{b}$ show the EP flux response (standardised). Colour bars represent each column. Stippling shows where the EM or ER response is significant ( $95 \%$ confidence interval, Methods). All data are for DJF.

Our proposed emergent constraint is therefore reasonably robust and is strongly linked to the physical processes. However, we note that other emergent constraints are possible. For example, ZWRI is correlated with the background SPV $(r=0.50, p=0.02$, Supplementary Fig. 1) but in this case the observations are near the middle of the model range so that $\mathrm{ER}$ is close to the simple ensemble mean. Had we only considered this relationship we would have erroneously constrained the response to be near the EM, highlighting the importance of basing constraints on the physical processes ${ }^{61,82}$. It is also possible that the response could be further enhanced by coupling with the ocean ${ }^{17,41,44}$, albeit with greater variability $^{79}$, and/or by the pattern of sea ice loss $36,57,58,83$, and could depend on the phase of the quasi-biennial oscillation ${ }^{84}$. However, initial results from a subset of models for PAMIP experiments that are designed to investigate the effects of coupling and the pattern of sea ice loss (Methods) do not show consistent differences in ZWRI across models compared to the standard experiments. This suggests that these effects may not be large, but results from more models are needed for further assessment, and could provide an important out of sample test ${ }^{82}$ of the emergent constraint proposed here.

\section{Discussion}

We have analysed the boreal winter atmospheric circulation response to future Arctic sea ice loss using a very large multimodel ensemble, comprising 16 different models and more than 3000 ensemble members with the same experimental protocol. We find a robust response in the troposphere, with a weakening of mid-latitude tropospheric westerly winds and an equatorward shift of the storm tracks, consistent with a negative phase of the NAO. However, the strength of the modelled response varies between models and is proportional to the strength of the eddy momentum feedback, enabling a constrained estimate of the realworld response to be obtained. Since all the models underestimate the observed eddy feedback strength, the real-world response is likely to be at the higher end of the model range. The stratosphere response is not consistent across the models and therefore not essential for determining the sign of the tropospheric circulation response to sea ice loss. However, the eddy feedback constraint indicates a robust weakening of the stratospheric polar vortex, suggesting that in the real world the stratosphere may play an active role that amplifies the surface response, consistent with other studies $30,33,36,64,66,74$. 
Our emergent constraint suggests that models may underestimate the response to sea ice loss and is consistent with other evidence that models underestimate the predictable fraction of NAO variability in seasonal ${ }^{85-87}$, interannual ${ }^{88}$, and decadal forecasts ${ }^{89-91}$, and in historical climate simulations ${ }^{92-94}$. This has been referred to as the signal-to-noise paradox ${ }^{95}$ since models are unexpectedly able to predict the real world better than they can predict one of their own ensemble members. We find that the eddy momentum feedback is underestimated in all of the models, suggesting that this could be a potential cause of this model error as previously suggested ${ }^{96}$. Possible reasons for underestimated eddy feedback include errors in wave propagation, imperfect representation of interactions with the mean flow, and unresolved waves, but further analysis is left for future work. We also note that our emergent constraint could potentially explain some of the model spread in jet shifts forced by a variety of factors, including changes in greenhouse gases, but this is beyond the scope of the present study.

Diagnosing the response to sea ice loss is not possible from observations alone since causality cannot be established ${ }^{61}$. Nevertheless, there is a perception that models and observations do not agree ${ }^{5}$. Our results suggest that model errors could be responsible for some of the apparent inconsistencies and that taking these into account yields a robust modelled response in the troposphere and stratosphere. However, it is important to note that the short observational record contains considerable sampling uncertainty 97,98 . For example, the period 1979 to 2012 suggests a strong link between autumn sea ice and winter atmospheric circulation and Eurasian temperature (Fig. 9a, c, e) consistent with previous studies $6,20-25$. If we extend the analysis period by adding the most recent 8 years of data (2013-2020), we find that the observed relationships between autumn sea ice extent and DJF NAO, SPV and Eurasian $\mathrm{T}$ indices are weaker in magnitude (Fig. 9b, d, f). This is consistent with recent evidence that the observed relationships are modest ${ }^{7}$ and intermittent ${ }^{66}$ and may not reflect a causal link with Arctic sea ice ${ }^{99-101}$.

Our model estimates are within, and therefore consistent with, the magnitudes of the observed relationships based on the extended period of record (1979-2020). However, the modelled response to sea ice loss remains relatively weak, amounting to $30 \%$ or less of the observed interannual standard deviation $(\sigma)$ of the NAO and SPV. Assuming linearity and given that the imposed reduction in DJF Arctic sea ice extent in our experiments is around $4 \sigma$, this implies that interannual Arctic sea ice variations account for less than $10 \%$ of the interannual variations in NAO and SPV, and are thus unlikely to drive large seasonal mean impacts in individual winters. Nevertheless, these values are similar in magnitude to changes in the NAO and SPV expected by the end of the century in response to increases in greenhouse gases $^{65,102,103}$, and will therefore impact long term projections.

\section{Methods}

Model experiments. We assess coordinated experiments from the Polar Amplification Model Intercomparison Project ${ }^{62}$ (PAMIP). PAMIP experiment 1.1 simulates the present day climate using global atmosphere models constrained at the surface by present day estimates of sea surface temperature (SST) and sea ice concentration (SIC). PAMIP experiment 1.6 is the same as 1.1 except that Arctic SIC is replaced with values expected with global temperatures 2 degrees Celsius warmer than pre-industrial conditions. Where sea ice is lost in 1.6 relative to 1.1, future SSTs are used; elsewhere the SSTs are the same in 1.6 and 1.1. By construction, the difference between 1.6 and 1.1 provides the simulated response to future Arctic sea ice loss and associated local changes in SST. Each experiment starts on 1st April 2000 and runs for 14 months. We analyse results from 16 models, each with at least 98 ensemble members (Table 1) and forced with the same SSTs and SICs. We also analyse a small subset of models to assess the effects of ocean-atmosphere coupling (PAMIP experiments 2.1 and 2.3, EC-EARTH3, HadGEM3-MM, NorESM2-LM) and regional sea ice changes (PAMIP experiments 3.1 and 3.2, CNRM-CM6-1, ECHAM6.3, EC-EARTH3, HadGEM3-MM). All data were re-gridded to the resolution of the coarsest model ( $3^{\circ}$ latitude by $3^{\circ}$ longitude) before comparison.
Observations. We use Eliassen-Palm (EP) fluxes from three reanalyses (ERAInterim, NCEP-NCAR and JRA-55) available from the Centre for Environmental Data Analysis ${ }^{81}$. Sea ice observations are taken from HadISST1.1 ${ }^{104}$. Sea level pressure, near-surface temperature and stratospheric wind observations are taken from the ERA5 reanalysis ${ }^{105}$

Indices. The North Atlantic Oscillation (NAO) index is calculated as the difference in mean sea level pressure between two small boxes located around the Azores $\left(28-20^{\circ} \mathrm{W}, 36-40^{\circ} \mathrm{N}\right)$ and Iceland $\left(25-16^{\circ} \mathrm{W}, 63-70^{\circ} \mathrm{N}\right)$ with the average over the whole time series removed to create anomalies ${ }^{88}$. To quantify the response to Arctic sea ice loss, we define a Zonal Wind Response Index (ZWRI) that is calculated as the difference in zonally averaged zonal wind response between $30-39^{\circ} \mathrm{N}$ and $54-63^{\circ} \mathrm{N}$ averaged over 600 to $150 \mathrm{hPa}$, which correspond to the regions with the largest zonal wind responses in the multi-model mean. The strength of the stratospheric polar vortex (SPV) is computed as the zonal-mean zonal wind averaged over $54-66^{\circ} \mathrm{N}$ at $10 \mathrm{hPa}$. Eurasian temperatures (Eurasia $\mathrm{T}$ ) are averaged over the region ${ }^{34} 60-120^{\circ} \mathrm{E}, 40-60^{\circ} \mathrm{N}$. The Barents-Kara (BK) Seas region ${ }^{58}$ is taken as $10-100^{\circ} \mathrm{E}, 65-85^{\circ} \mathrm{N}$.

Thermal wind. The geostrophic balance between pressure gradient and Coriolis forces, combined with the hydrostatic equation leads to the thermal wind relationship, showing that a reduction in meridional temperature gradient as the Arctic warms is accompanied by a decrease in vertical wind shear ${ }^{68}$ :

$$
f \frac{\partial u}{\partial p}=\frac{R}{a p} \frac{\partial T}{\partial \phi}
$$

where $u, T, p, \phi$ are zonal wind, temperature, pressure and latitude, $f$ is the Coriolis parameter, $a$ is the radius of the Earth and $R$ is the gas constant.

\section{Transformed Eulerian Mean momentum equation and Eliassen-Palm fluxes.} Much insight into extratropical atmospheric circulation can be gained from the Transformed Eulerian Mean (TEM) form of the zonal mean quasi-geostrophic (QG) momentum equation ${ }^{68,81}$ :

$$
\frac{\partial \bar{u}}{\partial t}=f \bar{v}^{*}+\frac{1}{a \cos \phi} \nabla \cdot \boldsymbol{F}+\bar{\epsilon}
$$

where the overbar represents the zonal mean and the $*$ denotes the TEM circulation:

$$
\begin{gathered}
\bar{v}^{*}=\bar{v}-\frac{\partial}{\partial p}\left[\frac{\overline{v^{\prime} \theta^{\prime}}}{\partial \bar{\theta} / \partial p}\right] \\
\bar{w}^{*}=\bar{w}+\frac{1}{a \cos \phi} \frac{\partial}{\partial \phi}\left[\frac{\overline{v^{\prime} \theta^{\prime}} \cos \phi}{\partial \bar{\theta} / \partial p}\right]
\end{gathered}
$$

where the prime represents departures from the zonal mean, $u, v, w$ are the zonal, meridional and vertical velocities, $\theta$ is potential temperature, $f$ is the Coriolis parameter, $t$ is time, $\bar{\epsilon}$ represents the effects of friction and parameterised processes, and the Eliassen-Palm (EP) flux divergence is given by

$$
\nabla \cdot \boldsymbol{F}=\nabla_{\phi} F_{\phi}+\nabla_{p} F_{p}=\frac{1}{a \cos \phi} \frac{\partial F_{\phi} \cos \phi}{\partial \phi}+\frac{\partial F_{p}}{\partial p}
$$

where the QG northward and vertical EP fluxes are given by

$$
\left\{F_{\phi}, F_{p}\right\}=a \cos \phi\left\{-\overline{u^{\prime} v^{\prime}}, \frac{\overline{v^{\prime} \theta^{\prime}}}{\partial \bar{\theta} / \partial p} f\right\}
$$

Note, that we use the full primitive equation EP fluxes ${ }^{81}$ in our analysis but the main effects are captured by the QG form given above.

Standardisation. To aid visualisation the TEM circulation and EP fluxes are scaled by dividing by the internal variability of the present-day simulations, computed as the variance across ensemble members, averaged over all of the models, and then square-rooted to obtain the standard deviation. This standardises the magnitude of signals across the depth of the atmosphere and indicates the general sense of the propagation (i.e. north or south, upwards or downwards) but does not necessarily indicate the precise geometric direction.

Refractive index. Insight into the propagation of wave activity can be gained by examining the refractive index ${ }^{106,107}$ :

$$
n_{k}^{2}=\frac{\bar{q}_{\phi}}{\bar{u}}-\left(\frac{k}{a \cos \phi}\right)^{2}-\left(\frac{f}{2 N H}\right)^{2}
$$

where

$$
\bar{q}_{\phi}=\frac{2 \Omega}{a} \cos \phi-\frac{1}{a^{2}}\left[\frac{(\bar{u} \cos \phi)_{\phi}}{\cos \phi}\right]_{\phi}-\frac{f^{2}}{\rho_{0}}\left(\rho_{0} \frac{\bar{u}_{z}}{N^{2}}\right)_{z}
$$


(a) NAO (1979-2012)

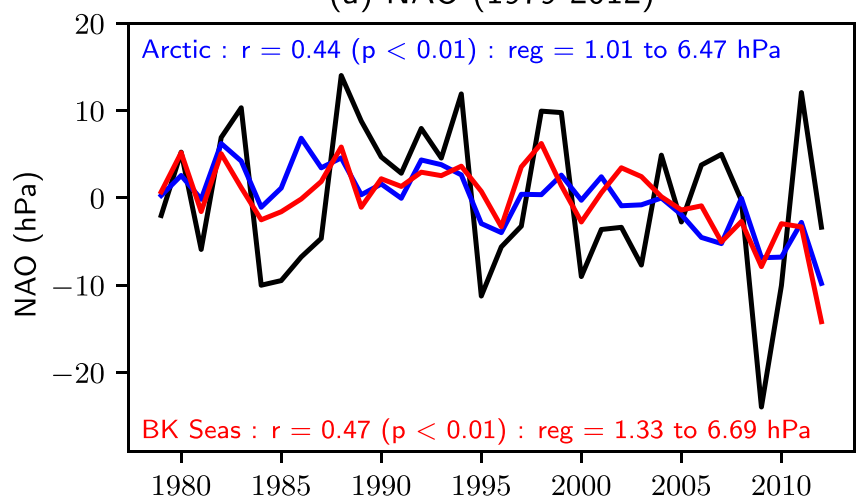

(c) SPV (1979-2012)

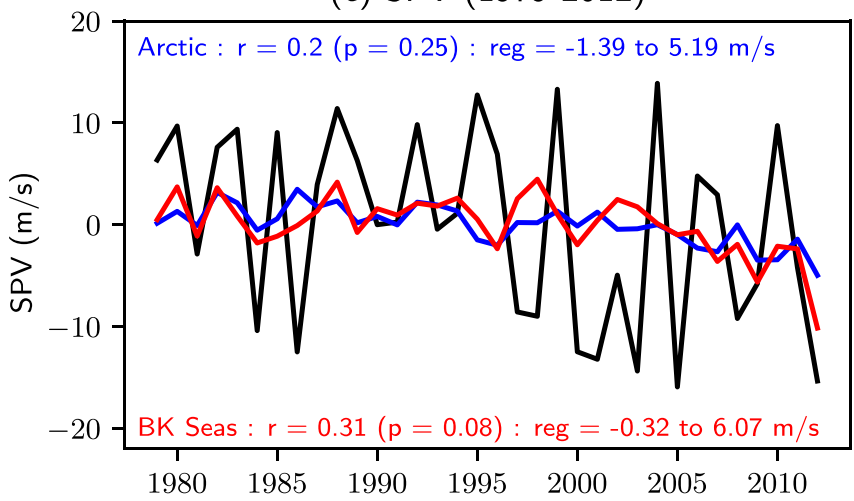

(e) Eurasia T (1979-2012)

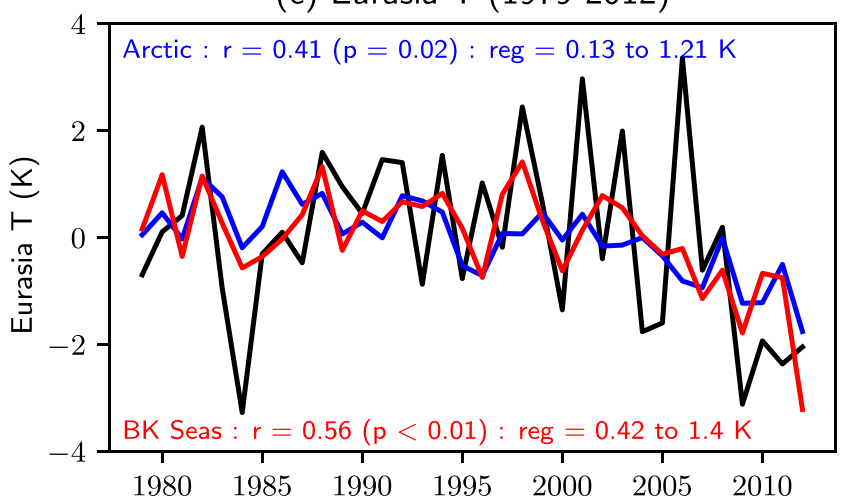

(b) NAO (1979-2020)

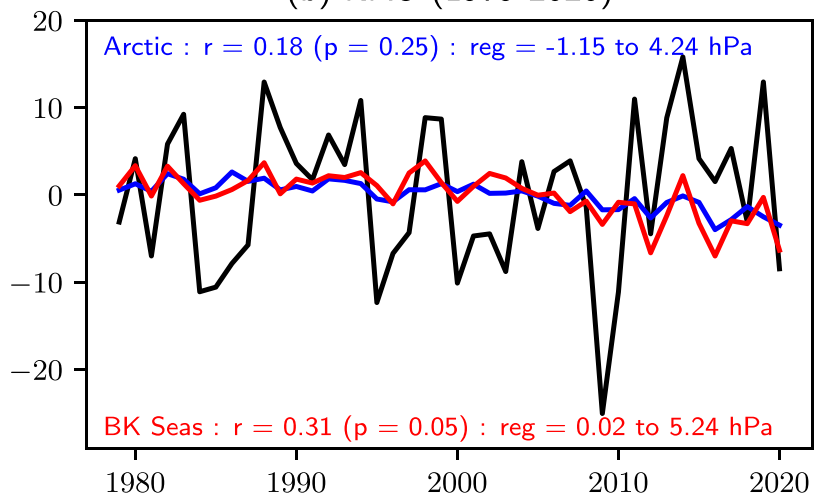

(d) SPV (1979-2020)

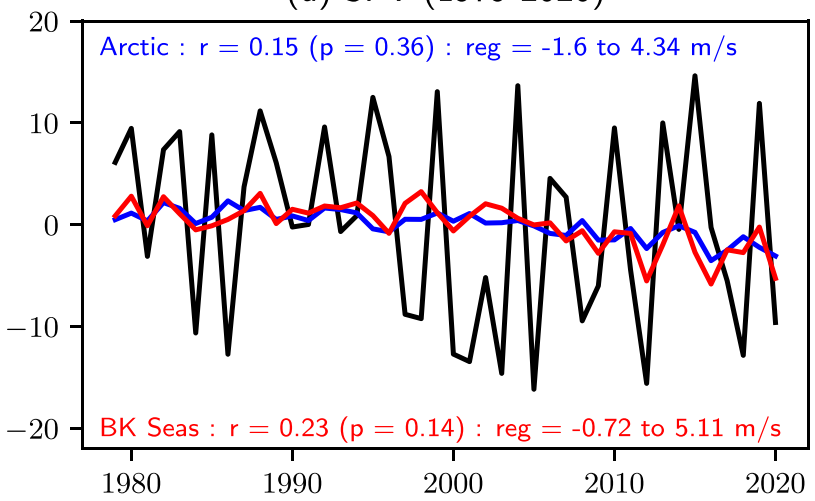

(f) Eurasia $T(1979-2020)$

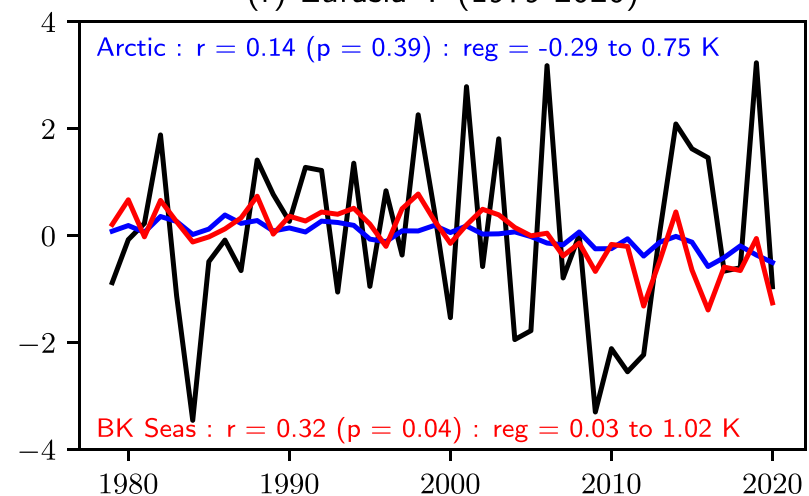

Fig. 9 Weakened observed relationships. a Observed winter (DJF) NAO anomaly time-series for the period 1979 to 2012 (black) along with the variability that is linearly related to autumn (September-November) sea ice extent in the Arctic (blue) and Barents-Kara (BK) Seas (red). Pearson correlation ( $r$ ) and regression coefficients (reg, 95\% confidence interval, per standard deviation of sea ice extent) are indicated. b As a but for the period 1979 to 2020. c, d As a, b but for SPV. e, f As a, b but for Eurasia T. Indices are defined in Methods.

is the meridional gradient of the zonal mean potential vorticity, and $k, N, H$ and $\Omega$ denote the zonal wave number, buoyancy frequency, scale height, and Earth rotation frequency respectively. Note, that waves are refracted towards high values of $n_{k}^{2}$.

Eddy feedback. Eddies are generated by baroclinic processes in the storm tracks and eddy activity propagates horizontally and vertically. Eddies flux angular momentum in the opposite direction to their propagation, driving (accelerating) the zonal wind in their source regions and dragging (decelerating) the zonal wind in their dissipation regions. These interactions with the mean flow can promote or reduce further eddy generation, producing a positive or negative eddy feedback ${ }^{77,78}$. In simple models, it is possible to switch off the effects of eddies allowing eddy feedback to be diagnosed by comparing to the full response ${ }^{108}$. Previous studies have assessed eddy feedback in comprehensive models using lagged relationships in daily data focussing on large scale patterns of variability ${ }^{109,110}$. However, lagged relationships potentially include persistence that is unrelated to feedbacks and may miss fast feedbacks that occur before the diagnosed lag. They also require analysing large volumes of daily data.

Here, we propose a new approach that can be used with seasonal mean data and does not require lags to be specified. We reason that the fraction of seasonal mean zonal wind variability that is related to eddies will increase as the eddy feedback becomes more positive, and measure the eddy feedback strength as the local correlation squared (i.e. the variance explained) between DJF $\bar{u}$ and $\nabla_{\phi} F_{\phi}$ averaged over the mid to upper northern troposphere $\left(25-72^{\circ} \mathrm{N}, 600-200 \mathrm{hPa}\right.$, the box shown in Fig. 6a). Although this measure is imperfect since it includes eddy driving, we show that it explains some of the differences in modelled responses. For the model simulations, the eddy feedback parameter is calculated across the ensemble members for the present-day simulations, whereas for the reanalyses it is calculated from time series covering the period 1979-2016. The reanalyses eddy feedback parameters are insensitive to removing a linear trend, but we find some sensitivity to the different ways of calculating eddy feedback using models for which time series data are also available (see main text). However, our main conclusions remain valid. 


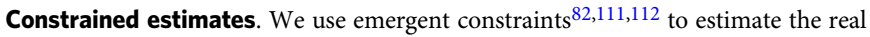
world response. We seek an observable quantity $(x)$ that provides a physical explanation for differences in the model estimates of the response to Arctic sea ice loss $\left(y_{i}\right.$, the ensemble mean estimate of the response for model $\left.i\right)$ such that

$$
y_{i}=\bar{y}_{i}+\beta x_{i}+\varepsilon_{i}
$$

where the overbar denotes the average of the model estimates, $\beta$ is the slope of the linear regression between $x_{i}$ and $y_{i}$, and $\varepsilon_{i}$ is an identically independently distributed random variable with zero expectation (i.e. noise).

If such a quantity exists, then the simple multi-model ensemble mean (EM i.e. with $\beta=0$ ) is an inappropriate estimate of the true response because $\varepsilon_{i}$ would not be independent of $x^{112}$. Instead, the observable response $\left(y_{O}\right)$ may be estimated using ensemble regression ${ }^{112}$ (ER):

$$
y_{O}=\bar{y}_{i}+\beta\left(x_{O}-\bar{x}_{i}\right)
$$

where $x_{O}$ is the observed estimate of $x$. The error variance of the multi-model ensemble mean is

$$
S_{y}^{2}=\sigma_{\varepsilon}^{2}\left(\frac{1}{n}+\frac{\left(x_{O}-\bar{x}_{i}\right)^{2}}{\sum_{i=1}^{n}\left(x_{i}-\bar{x}_{i}\right)}\right)
$$

where $n$ is the number of models and $\sigma_{\varepsilon}^{2}$ is the variance of $\varepsilon_{i}$. The second term in the parentheses accounts for estimation error in regression slope and grows quadratically with the error in the model mean estimate of $x$.

\section{Data availability}

PAMIP datasets analysed during the current study are available from the CMIP data archive https://esgf-node.llnl.gov/projects/cmip6/. Data for HadGEM3-LL are available from https://zenodo.org/record/5127891, and data for OpenIFS are available from https://cera-www.dkrz.de/WDCC/ui/cerasearch datasets DKRZ_LTA_995_ds00003 to DKRZ_LTA_995_ds00008 inclusive. Reanalyses EP fluxes are available from the Centre for Environmental Data Analysis https://catalogue.ceda.ac.uk/uuid/dafbd838e4cc4c68a 5ccdd90690ea57f. HadISST1.1 sea ice observations are available from https://www. metoffice.gov.uk/hadobs/hadisst/. ERA5 reanalysis data are available from https:// www.ecmwf.int/en/forecasts/datasets/reanalysis-datasets/era5.

\section{Code availability}

The code used during the current study is available from the corresponding author on reasonable request.

Received: 26 May 2021; Accepted: 17 January 2022;

Published online: 07 February 2022

\section{References}

1. Screen, J. A. \& Simmonds, I. The central role of diminishing sea ice in recent arctic temperature amplification. Nature 464, 1334-1337 (2010).

2. Vaughan, D. G. et al. Observations: Cryosphere. In Stocker, T. F. et al. (eds.) Climate Change 2013: The Physical Science Basis. Contribution of Working Group I to the Fifth Assessment Report of the Intergovernmental Panel on Climate Change (Cambridge University Press, 2013).

3. Collins, M. et al. Long-term climate change: projections, commitments and irreversibility. In Stocker, T. F. et al. (eds.) Climate Change 2013: The Physical Science Basis. Contribution of Working Group I to the Fifth Assessment Report of the Intergovernmental Panel on Climate Change, 1029-1136 (Cambridge University Press, 2013).

4. Cohen, J. et al. Recent Arctic amplification and extreme mid-latitude weather. Nat. Geosci. 7, 627-637 (2014)

5. Cohen, J. et al. Divergent consensuses on Arctic amplification influence on midlatitude severe winter weather. Nat. Clim. Change 10, 20-29 (2020).

6. Overland, J. et al. The Melting Arctic and Midlatitude weather patterns: are they connected? J. Clim. 28, 7917-7932 (2015).

7. Blackport, R. \& Screen, J. A. Weakened evidence for mid-latitude impacts of Arctic warming. Nat. Clim. Change 10, 1065-1066 (2020).

8. Francis, J. A. \& Vavrus, S. J. Evidence linking Arctic amplification to extreme weather in mid-latitudes. Geophys. Res. Lett. 39, L06801 (2012).

9. Wallace, J. M., Held, I. M., Thompson, D. W. J., Trenberth, K. E. \& Walsh, J. E. Global warming and winter weather. Science 343, 729-730 (2014).

10. Kintisch, E. Into the Maelstrom. Science 344, 250-253 (2014).

11. Vihma, T. Effects of Arctic Sea Ice decline on weather and climate: a review. Surv. Geophysics 35, 1175-1214 (2014).

12. Walsh, J. E. Intensified warming of the Arctic: causes and impacts on middle latitudes. Glob. Planet. Change 117, 52-63 (2014).

13. Barnes, E. A. \& Screen, J. A. The impact of Arctic warming on the midlatitude jet-stream: Can it? Has it? Will it? Wiley Interdiscip. Rev.: Clim. Change 6, 277-286 (2015).
14. Gramling, C. Arctic impact. Science 347, 818-821 (2015).

15. Shepherd, T. G. Effects of a warming Arctic. Science 353, 989-990 (2016)

16. McCusker, K. E., Fyfe, J. C. \& Sigmond, M. Twenty-five winters of unexpected Eurasian cooling unlikely due to Arctic sea-ice loss. Nat. Geosci. 9, 838-842 (2016).

17. Screen, J. A. et al. Consistency and discrepancy in the atmospheric response to Arctic sea-ice loss across climate models. Nat. Geosci. 11, 155-163 (2018).

18. Blackport, R., Screen, J. A., van der Wiel, K. \& Bintanja, R. Minimal influence of reduced Arctic sea ice on coincident cold winters in mid-latitudes. Nat. Clim. Change 9, 697-704 (2019).

19. Dai, A. \& Song, M. Little influence of Arctic amplification on mid-latitude climate. Nat. Clim. Change 10, 231-237 (2020).

20. Outten, S. D. \& Esau, I. A link between Arctic sea ice and recent cooling trends over Eurasia. Climatic Change 110, 1069-1075 (2012).

21. Inoue, J., Hori, M. E. \& Takaya, K. The role of Barents Sea Ice in the wintertime cyclone track and emergence of a warm-arctic cold-siberian anomaly. J. Clim. 25, 2561-2568 (2012).

22. Liu, J., Curry, J. A., Wang, H., Song, M. \& Horton, R. M. Impact of declining Arctic sea ice on winter snowfall. Proc. Natl Acad. Sci. USA 109, 4074-4079 (2012).

23. García-Serrano, J., Frankignoul, C., Gastineau, G. \& de la Cámara, A. On the predictability of the winter euro-atlantic climate: lagged influence of autumn Arctic Sea Ice. J. Clim. 28, 5195-5216 (2015).

24. Kug, J.-S. et al. Two distinct influences of Arctic warming on cold winters over North America and East Asia. Nat. Geosci. 8, 759-762 (2015).

25. King, M. P., Hell, M. \& Keenlyside, N. Investigation of the atmospheric mechanisms related to the autumn sea ice and winter circulation link in the Northern Hemisphere. Clim. Dyn. 46, 1185-1195 (2016).

26. Simon, A., Frankignoul, C., Gastineau, G. \& Kwon, Y.-O. An observational estimate of the direct response of the cold-season atmospheric circulation to the Arctic Sea Ice Loss. J. Clim. 33, 3863-3882 (2020).

27. Honda, M., Inoue, J. \& Yamane, S. Influence of low Arctic seaice minima on anomalously cold Eurasian winters. Geophys. Res. Lett. 36, L08707 (2009).

28. Petoukhov, V. \& Semenov, V. A. A link between reduced Barents-Kara sea ice and cold winter extremes over northern continents. J. Geophys. Res. 115, D21111 (2010).

29. Mori, M., Watanabe, M., Shiogama, H., Inoue, J. \& Kimoto, M. Robust Arctic sea-ice influence on the frequent Eurasian cold winters in past decades. Nat. Geosci. 7, 869-873 (2014).

30. Kim, B.-M. et al. Weakening of the stratospheric polar vortex by Arctic sea-ice loss. Nat. Commun. 5, 4646 (2014).

31. Peings, Y. \& Magnusdottir, G. Response of the wintertime northern hemisphere atmospheric circulation to current and projected arctic sea ice decline: a numerical study with CAM5. J. Clim. 27, 244-264 (2014).

32. Nakamura, T. et al. A negative phase shift of the winter AO/NAO due to the recent Arctic seaice reduction in late autumn. J. Geophys. Res.: Atmospheres 120, 3209-3227 (2015).

33. Zhang, P. et al. A stratospheric pathway linking a colder Siberia to BarentsKara Sea sea ice loss. Sci. Adv. 4, eaat6025 (2018).

34. Mori, M., Kosaka, Y., Watanabe, M., Nakamura, H. \& Kimoto, M. A reconciled estimate of the influence of Arctic sea-ice loss on recent Eurasian cooling. Nat. Clim. Change 9, 123-129 (2019).

35. Deser, C., Tomas, R., Alexander, M. \& Lawrence, D. The seasonal atmospheric response to projected arctic sea ice loss in the late twenty-first century. J. Clim. 23, 333-351 (2010).

36. Sun, L., Deser, C. \& Tomas, R. A. Mechanisms of stratospheric and tropospheric circulation response to projected arctic sea ice loss. J. Clim. 28, 7824-7845 (2015)

37. Ayarzagüena, B. \& Screen, J. A. Future Arctic sea ice loss reduces severity of cold air outbreaks in midlatitudes. Geophys. Res. Lett. 43, 2801-2809 (2016).

38. Screen, J. A. The missing Northern European winter cooling response to Arctic sea ice loss. Nat. Commun. 8, 14603 (2017)

39. Ogawa, F. et al. Evaluating impacts of recent Arctic Sea ice loss on the northern hemisphere winter climate change. Geophys. Res. Lett. 45, 3255-3263 (2018).

40. He, B. et al. CAS FGOALS-f3-L large-ensemble simulations for the CMIP6 polar amplification model intercomparison project. Adv. Atmos. Sci. 38, 1028-1049 (2021)

41. Deser, C., Sun, L., Tomas, R. A. \& Screen, J. Does ocean coupling matter for the northern extratropical response to projected Arctic sea ice loss? Geophys. Res. Lett. 43, 2149-2157 (2016).

42. Chripko, S. et al. Impact of reduced arctic sea ice on northern hemisphere climate and weather in autumn and winter. J. Clim. 1-61 (2021).

43. Seierstad, I. A. \& Bader, J. Impact of a projected future Arctic Sea Ice reduction on extratropical storminess and the NAO. Clim. Dyn. 33, 937-943 (2009).

44. Deser, C., Tomas, R. A. \& Sun, L. The role of ocean-atmosphere coupling in the zonal-mean atmospheric response to Arctic Sea ice loss. J. Clim. 28, $2168-2186$ (2015). 
45. Singarayer, J. S., Bamber, J. L. \& Valdes, P. J. Twenty-first-century climate impacts from a declining Arctic Sea ice cover. J. Clim. 19, 1109-1125 (2006)

46. Strey, S. T., Chapman, W. L. \& Walsh, J. E. The 2007 sea ice minimum: impacts on the Northern Hemisphere atmosphere in late autumn and early winter. J. Geophys. Res. 115, D23103 (2010).

47. Orsolini, Y. J., Senan, R., Benestad, R. E. \& Melsom, A. Autumn atmospheric response to the 2007 low Arctic sea ice extent in coupled ocean-atmosphere hindcasts. Clim. Dyn. 38, 2437-2448 (2012).

48. Rinke, A., Dethloff, K., Dorn, W., Handorf, D. \& Moore, J. C. Simulated Arctic atmospheric feedbacks associated with late summer sea ice anomalies: atmospheric feedbacks to arctic sea ice. J. Geophys. Res.: Atmosph. 118, 7698-7714 (2013).

49. Cassano, E. N., Cassano, J. J., Higgins, M. E. \& Serreze, M. C. Atmospheric impacts of an Arctic sea ice minimum as seen in the Community Atmosphere Model. Int. J. Climatol. 34, 766-779 (2014).

50. Screen, J. A., Deser, C., Simmonds, I. \& Tomas, R. Atmospheric impacts of Arctic sea-ice loss, 1979-2009: separating forced change from atmospheric internal variability. Clim. Dyn. 43, 333-344 (2014).

51. Screen, J. A., Simmonds, I., Deser, C. \& Tomas, R. The atmospheric response to three decades of observed arctic sea ice loss. J. Clim. 26, 1230-1248 (2013).

52. Petrie, R. E., Shaffrey, L. C. \& Sutton, R. T. Atmospheric impact of arctic sea ice loss in a coupled ocean-atmosphere simulation. J. Clim. 28, 9606-9622 (2015).

53. Blackport, R. \& Kushner, P. J. The transient and equilibrium climate response to rapid summertime sea ice loss in CCSM4. J. Clim. 29, 401-417 (2016)

54. Semmler, T. et al. Seasonal atmospheric responses to reduced arctic sea ice in an ensemble of coupled model simulations. J. Clim. 29, 5893-5913 (2016).

55. Pedersen, R. A., Cvijanovic, I., Langen, P. L. \& Vinther, B. M. The impact of regional arctic sea ice loss on atmospheric circulation and the NAO. J. Clim. 29, 889-902 (2016).

56. Chen, H. W., Zhang, F. \& Alley, R. B. The robustness of midlatitude weather pattern changes due to Arctic Sea ice loss. J. Clim. 29, 7831-7849 (2016).

57. McKenna, C. M., Bracegirdle, T. J., Shuckburgh, E. F., Haynes, P. H. \& Joshi, M. M. Arctic Sea Ice loss in different regions leads to contrasting northern hemisphere impacts. Geophys. Res. Lett. 45, 945-954 (2018).

58. Screen, J. A. Simulated atmospheric response to regional and pan-arctic sea ice loss. J. Clim. 30, 3945-3962 (2017).

59. Balmaseda, M. A., Ferranti, L., Molteni, F. \& Palmer, T. N. Impact of 2007 and 2008 Arctic ice anomalies on the atmospheric circulation: Implications for long-range predictions. Q. J. R. Meteorological Soc. 136, 1655-1664 (2010).

60. Screen, J. A. \& Francis, J. A. Contribution of sea-ice loss to Arctic amplification is regulated by Pacific Ocean decadal variability. Nat. Clim. Change 6, 856-860 (2016).

61. Smith, D. M. et al. Atmospheric Response to Arctic and Antarctic Sea Ice: the importance of ocean-atmosphere coupling and the background state. J. Clim. 30, 4547-4565 (2017).

62. Smith, D. M. et al. The Polar Amplification Model Intercomparison Project (PAMIP) contribution to CMIP6: investigating the causes and consequences of polar amplification. Geosci. Model Dev. 12, 1139-1164 (2019).

63. Eyring, V. et al. Overview of the coupled model intercomparison project phase 6 (CMIP6) experimental design and organisation. Geosci. Model Devel. 8 , 10539-10583 (2016).

64. Kretschmer, M. et al. More-persistent weak stratospheric polar vortex states linked to cold extremes. Bull. Am. Meteorological Soc. 99, 49-60 (2018).

65. Kretschmer, M., Zappa, G. \& Shepherd, T. G. The role of Barents-Kara sea ice loss in projected polar vortex changes. Weather Clim. Dyn. 1, 715-730 (2020).

66. Siew, P. Y. F., Li, C., Sobolowski, S. P. \& King, M. P. Intermittency of Arcticmid-latitude teleconnections: stratospheric pathway between autumn sea ice and the winter North Atlantic Oscillation. Weather Clim. Dyn. 1, 261-275 (2020).

67. Hell, M. C., Schneider, T. \& Li, C. Atmospheric circulation response to shortterm arctic warming in an idealized model. J. Atmos. Sci. 77, 531-549 (2020).

68. Vallis, G. K. Atmospheric and Oceanic Fluid Dynamics. (Cambridge University Press, Cambridge, 2017). OCLC: 1007089387.

69. Jaiser, R., Dethloff, K. \& Handorf, D. Stratospheric response to Arctic sea ice retreat and associated planetary wave propagation changes. Tellus A: Dyn. Meteorol. Oceanogr. 65, 19375 (2013).

70. Feldstein, S. B. \& Lee, S. Intraseasonal and interdecadal jet shifts in the northern hemisphere: the role of warm pool tropical convection and sea ice. J. Clim. 27, 6497-6518 (2014).

71. Overland, J. E. et al. Nonlinear response of mid-latitude weather to the changing Arctic. Nat. Clim. Change 6, 992-999 (2016).

72. Butler, A. H., Thompson, D. W. J. \& Heikes, R. The steady-state atmospheric circulation response to climate change-like thermal forcings in a simple general circulation model. J. Clim. 23, 3474-3496 (2010).

73. Shaw, T. A. et al. Storm track processes and the opposing influences of climate change. Nat. Geosci. 9, 656-664 (2016).
74. Wu, Y. \& Smith, K. L. Response of northern hemisphere midlatitude circulation to arctic amplification in a simple atmospheric general circulation model. J. Clim. 29, 2041-2058 (2016).

75. Shaw, T. A. Mechanisms of future predicted changes in the zonal mean midlatitude circulation. Curr. Clim. Change Rep. 5, 345-357 (2019).

76. Kidston, J. et al. Stratospheric influence on tropospheric jet streams, storm tracks and surface weather. Nat. Geosci. 8, 433-440 (2015).

77. Robinson, W. A. On the self-maintenance of midlatitude jets. J. Atmos. Sci. 63, 2109-2122 (2006)

78. Gerber, E. P. \& Vallis, G. K. Eddy-zonal flow interactions and the persistence of the zonal index. J. Atmos. Sci. 64, 3296-3311 (2007).

79. Peings, Y., Labe, Z. M. \& Magnusdottir, G. Are 100 ensemble members enough to capture the remote atmospheric response to $+2^{\circ} \mathrm{C}$ Arctic Sea Ice Loss? J. Clim. 34, 3751-3769 (2021).

80. Brient, F. \& Schneider, T. Constraints on climate sensitivity from space-based measurements of low-cloud reflection. J. Clim. 29, 5821-5835 (2016).

81. Martineau, P., Wright, J. S., Zhu, N. \& Fujiwara, M. Zonal-mean data set of global atmospheric reanalyses on pressure levels. Earth Syst. Sci. Data 10, 1925-1941 (2018).

82. Hall, A., Cox, P., Huntingford, C. \& Klein, S. Progressing emergent constraints on future climate change. Nat. Clim. Change 9, 269-278 (2019).

83. Levine, X. J., Cvijanovic, I., Ortega, P., Donat, M. G. \& Tourigny, E. Atmospheric feedback explains disparate climate response to regional Arctic sea-ice loss. npj Clim. Atmos. Sci. 4, 28 (2021)

84. Labe, Z., Peings, Y. \& Magnusdottir, G. The effect of QBO phase on the atmospheric response to projected Arctic Sea Ice Loss in Early Winter. Geophys. Res. Lett. 46, 7663-7671 (2019).

85. Eade, R. et al. Do seasonal-to-decadal climate predictions underestimate the predictability of the real world? Geophys. Res. Lett. 41, 5620-5628 (2014).

86. Scaife, A. A. et al. Skillful long-range prediction of european and north american winters. Geophys. Res. Lett. 41, 2514-2519 (2014).

87. Baker, L. H., Shaffrey, L. C., Sutton, R. T., Weisheimer, A. \& Scaife, A. A. An intercomparison of skill and over/underconfidence of the wintertime North Atlantic Oscillation in multi-model seasonal forecasts. Geophys. Res. Lett. 45, 7808-7817 (2018).

88. Dunstone, N. J. et al. Skilful predictions of the winter North Atlantic Oscillation one year ahead. Nature Geosci. 9, 809-814 (2016).

89. Athanasiadis, P. J. et al. Decadal predictability of North Atlantic blocking and the NAO. npj Clim. Atmos. Sci. 3, 20 (2020).

90. Smith, D. M. et al. North Atlantic climate far more predictable than models imply. Nature 583, 796-800 (2020).

91. Zhang, W., Kirtman, B., Siqueira, L., Clement, A. \& Xia, J. Understanding the signal-to-noise paradox in decadal climate predictability from CMIP5 and an eddying global coupled model. Clim. Dyn. 56, 2895-2913 (2021).

92. Zhang, W. \& Kirtman, B. Understanding the signal-to-noise paradox with a simple Markov model. Geophys. Res. Lett. 46, 13308-13317 (2019).

93. Sévellec, F. \& Drijfhout, S. S. The signal-to-noise paradox for interannual surface atmospheric temperature predictions. Geophys. Res. Lett. 46, 9031-9041 (2019)

94. Klavans, J. M., Cane, M. A., Clement, A. C. \& Murphy, L. N. NAO predictability from external forcing in the late 20th century. npj Clim. Atmos. Sci. 4, 22 (2021).

95. Scaife, A. A. \& Smith, D. A signal-to-noise paradox in climate science. $n p j$ Clim. Atmos. Sci. 1, 28 (2018).

96. Scaife, A. A. et al. Does increased atmospheric resolution improve seasonal climate predictions? Atmos. Sci. Lett. 20, e922 (2019).

97. Kolstad, E. W. \& Screen, J. A. Nonstationary relationship between Autumn Arctic Sea Ice and the Winter North Atlantic Oscillation. Geophys. Res. Lett. 46, 7583-7591 (2019).

98. Warner, J. L., Screen, J. A. \& Scaife, A. A. Links between Barents-Kara Sea Ice and the extratropical atmospheric circulation explained by internal variability and tropical forcing. Geophys. Res. Lett. 47, e2019GL085679 (2020).

99. Peings, Y. Ural blocking as a driver of early-winter stratospheric warmings. Geophys. Res. Lett. 46, 5460-5468 (2019).

100. He, S., Xu, X., Furevik, T. \& Gao, Y. Eurasian cooling linked to the vertical distribution of arctic warming. Geophys. Res. Lett. 47, e2020GL087212 (2020).

101. Labe, Z., Peings, Y. \& Magnusdottir, G. Warm Arctic, Cold Siberia Pattern: Role of Full Arctic Amplification Versus Sea Ice Loss Alone. Geophys. Res. Lett. 47, e2020GL088583 (2020).

102. Christensen, J. et al. Climate phenomena and their relevance for future regional climate change. (Cambridge University Press, New York, 2013).

103. Wu, Y., Simpson, I. R. \& Seager, R. Intermodel spread in the northern hemisphere stratospheric polar vortex response to climate change in the CMIP5 models. Geophys. Res. Lett. 46, 13290-13298 (2019).

104. Rayner, N. A. et al. Global analyses of SST, sea ice and night marine air temperature since the late nineteenth century. J. Geophys. Res. 108, 4407 (2003). 
105. Hersbach, H. et al. The ERA5 global reanalysis. Q. J. R. Meteorol. Soc. 146, 1999-2049 (2020).

106. Matsuno, T. Vertical propagation of stationary planetary waves in the winter northern hemisphere. J. Atmos. Sci. 27, 871-883 (1970).

107. Li, Q., Graf, H.-F. \& Giorgetta, M. A. Stationary planetary wave propagation in Northern Hemisphere winter - climatological analysis of the refractive index. Atmos. Chem. Phys. 7, 183-200 (2007).

108. Ronalds, B. \& Barnes, E. A. A role for barotropic eddy-mean flow feedbacks in the zonal wind response to sea ice loss and arctic amplification. J. Clim. 32, 7469-7481 (2019).

109. Lorenz, D. J. \& Hartmann, D. L. Eddy-zonal flow feedback in the northern hemisphere winter. J. Clim. 16, 1212-1227 (2003).

110. Hitchcock, P. \& Simpson, I. R. Quantifying eddy feedbacks and forcings in the tropospheric response to stratospheric sudden warmings. J. Atmos. Sci. 73, 3641-3657 (2016).

111. Hall, A. \& Qu, X. Using the current seasonal cycle to constrain snow albedo feedback in future climate change. Geophys. Res. Lett. 33, L03502 (2006).

112. Bracegirdle, T. J. \& Stephenson, D. B. Higher precision estimates of regional polar warming by ensemble regression of climate model projections. Clim. Dyn. 39, 2805-2821 (2012).

\section{Acknowledgements}

D.M.S., R.E., L.H., L.S.G., T.J., T.S., X.L., and P.O. were supported by the EU H2020 APPLICATE project (GA727862). The Met Office contribution was also supported by the Met Office Hadley Centre Climate Programme funded by BEIS and Defra and by the UK-China Research and Innovation Partnership Fund through the Met Office Climate Science for Service Partnership (CSSP) China as part of the Newton Fund. J.A.S was supported by NERC grants NE/P006760/1, NE/R005125/1 and NE/V005855/1. G.M and Y.P. were supported by the US Department of Energy, grant number DE-SC0019407. L.S.G was also supported by the Research council of Norway INES project (270061), and the Norwegian e-infrastructure for Research and Education (UNINETT Sigma2) through projects NN2345K, NS2345K and NS9034K. E.M. and D.M. acknowledge the support of the German Federal Ministry of Education and Research through the JPI Climate/JPI Oceans NextG-Climate Science-ROADMAP (FKZ: 01LP2002A) project and of the European Union's Horizon 2020 Programme through the Blue-Action Project (GA727852); and the use of resources from the DKRZ bm0966 and bm1190 projects. C. Deser acknowledges support from the National Center for Atmospheric Research, which is a major facility sponsored by the US National Science Foundation under cooperative agreement 1852977 . M.M. was supported by MEXT through the Integrated Research Program for Advancing Climate Models (JPMXD0717935457) and ArCS II (JPMXD1420318865) programs, and by the Environment Research and Technology Development Fund (JPMEERF20192004). J.G.-S. and P.O. were supported by the Spanish Ramón y Cajal' programme (RYC-2016-21181, RYC-2016-22772). B.H. was jointly funded by the Strategic Priority Research Program of the Chinese Academy of Sciences (Grant No. XDA19070404) and the National Natural Science Foundation of China (Grant Nos. 42030602, 91837101). G.G. was supported by the EU H2020
Blue-Action (GA727852) project and uses the HPC resources of TGCC under the allocations 2018-R0040110492 and 2019-A0060107732 made by GENCI. J.S. acknowledges the project L4 of the Collaborative Research Centre TRR 181 Energy Transfers in Atmosphere and Ocean funded by the Deutsche Forschungsgemeinschaft (DFG, German Research Foundation) under Project 274762653.

\section{Author contributions}

D.M.S. led the analysis and writing, R.E. analysed the data. M.B.A., H.A., A.C., S.C., C.D. N.J.D., J.G.S., G.G., L.S.G., S.C.H., B.H., L.H., T.J., J.K., X.L., G.M., E.M., D.M., M.M., R.M., P.O., Y.P., A.A.S., J.A.S., T.S., M.Sig, J.S., L.S., A.W., D.M.S., and R.E. contributed to producing the model results. M.Sea contributed to producing the observed results. All authors commented on the manuscript.

\section{Competing interests}

The authors declare no competing interests.

\section{Additional information}

Supplementary information The online version contains supplementary material available at https://doi.org/10.1038/s41467-022-28283-y.

Correspondence and requests for materials should be addressed to D. M. Smith.

Peer review information Nature Communications thanks the anonymous reviewers for their contribution to the peer review of this work. Peer reviewer reports are available.

Reprints and permission information is available at http://www.nature.com/reprints

Publisher's note Springer Nature remains neutral with regard to jurisdictional claims in published maps and institutional affiliations.

(c) (i) Open Access This article is licensed under a Creative Commons Attribution 4.0 International License, which permits use, sharing, adaptation, distribution and reproduction in any medium or format, as long as you give appropriate credit to the original author(s) and the source, provide a link to the Creative Commons license, and indicate if changes were made. The images or other third party material in this article are included in the article's Creative Commons license, unless indicated otherwise in a credit line to the material. If material is not included in the article's Creative Commons license and your intended use is not permitted by statutory regulation or exceeds the permitted use, you will need to obtain permission directly from the copyright holder. To view a copy of this license, visit http://creativecommons.org/ licenses/by/4.0/.

(c) Crown 2022 